TITLE:

\title{
Thermo-reversible supramolecular hydrogels of trehalose-type diblock methylcellulose analogues
}

\section{$\operatorname{AUTHOR}(S)$ :}

Yamagami, Mao; Kamitakahara, Hiroshi; Yoshinaga, Arata; Takano, Toshiyuki

\section{CITATION:}

Yamagami, Mao ...[et al]. Thermo-reversible su pramolecular hydrogels of trehalose-type diblock methylcellulose analogues. Carbohydrate Polymers 2018, 183: 110-122

\section{ISSUE DATE:}

2018-03-01

URL:

http://hdl.handle.net/2433/231416

\section{RIGHT:}

(c) 2018. This manuscript version is made available under the CC-BY-NC-ND 4.0 license

http://creativecommons.org/licenses/by-nc-nd/4.0/. The full-text file will be made open to the public on 1 March 2019 in accordance with publisher's 'Terms and Conditions for Self-Archiving'. This is not the published version. Please cite only the published version.この論文は出版社版でありません。引用の際には出版社版をご確認ご利用ください。 
1 Thermo-reversible supramolecular hydrogels of trehalose-type diblock

2 methylcellulose analogues

Mao YAMAGAMI, Hiroshi KAMITAKAHARA, Arata YOSHINAGA, Toshiyuki TAKANO

5 Graduate School of Agriculture, Kyoto University, Sakyo-ku, Kyoto 606-8502, Japan

6 Corresponding Author: hkamitan@kais.kyoto-u.ac.jp

7 Phone: $+81-75-753-6257$

8 Fax: $+81-75-753-6300$

10 ABSTRACT:

11 This paper describes the design and synthesis of new trehalose-type diblock methylcellulose

12 analogues with nonionic, cationic, and anionic cellobiosyl segments, namely

13 1-(tri- $O$-methyl-cellulosyl)-4-[ $\beta$-D-glucopyranosyl-( $1 \rightarrow 4)$ - $\beta$-D-glucopyranosyloxymethyl]-1 $H$-1,2,3

14 -triazole (1), 1-(tri- $O$-methyl-cellulosyl)-4-[(6-amino-6-deoxy- $\beta$-D-glucopyranosyl)-( $1 \rightarrow 4)$ -

15 6-amino-6-deoxy- $\beta$-D-glucopyranosyloxymethyl]-1 $H$-1,2,3-triazole (2), and

16 4-(tri- $O$-methyl-cellulosyloxymethyl)-1-[ $\beta$-D-glucopyranuronosyl-( $1 \rightarrow 4)$ - $\beta$-D-glucopyranuronosyl]-

$171 H$-1,2,3-triazole (3), respectively. Aqueous solutions of all of the 1,2,3-triazole-linked diblock

18 methylcellulose analogues possessed higher surface activities than that of industrially produced

19 methylcellulose and exhibited lower critical solution temperatures, that allowed the formation of

20 thermoresponsive supramolecular hydrogels at close to human body temperature. Supramolecular

21 structures of thermo-reversible hydrogels based on compounds 1, 2, and 3 were investigated by

22 means of scanning electron microscopy (SEM) and transmission electron microscopy (TEM).

23 Detailed structure-property-function relationships of compounds 1, 2, and 3 were discussed. Not

24 only nonionic hydrophilic segment but also ionic hydrophilic segments of diblock methylcellulose

25 analogues were valid for the formation of thermo-reversible supramolecular hydrogels based on

26 end-functionalized methylcellulose.

28 Keywords: methylcellulose; polysaccharides; diblock copolymer; end-functionalization; surface 29 activity; thermo-reversible supramolecular hydrogels. 


\section{Introduction}

32

33

Methylcellulose (MC) is one of the more common cellulose ethers and has been of particular interest for the investigation of its structure-property relationships, such as the surface activity of its aqueous solution and its thermo-reversible gelation properties at elevated temperature. These properties of industrial and academic interest are attributed to the chemical structure of the methylcellulose skeleton. Therefore, many researchers have previously investigated MC. Commercial MC prepared under heterogeneous conditions is an alternating block copolymer composed of densely substituted hydrophobic and less densely substituted hydrophilic block sequences (Savage, 1957). The highly methylated region-a sequence of 2,3,6-tri-O-methyl-glucosyl residues - of the cellulose skeleton is said to cause micelles, that is, liquid-liquid phase separations in aqueous solution (Rees, 1972). These micelles are known as “crosslinking loci” (Kato, Yokoyama, \& Takahashi, 1978). In addition, it is well known that reversible crosslinks must exist in any reversible gel (Kato et al., 1978).

We have reported diblock methylcellulose derivatives with regioselective functionalization patterns (Nakagawa, Fenn, Koschella, Heinze, \& Kamitakahara, 2011b). We found direct evidence that a sequence of 2,3,6-tri- $O$-methyl-glucopyranosyl units causes thermo-reversible gelation of aqueous $\mathrm{MC}$ solution and that an idealized diblock structure consisting of 2,3,6-tri- $O$-methyl-glucopyranosyl and unmodified cello-oligosaccharides caused gelation (Nakagawa, Fenn, Koschella, Heinze, \& Kamitakahara, 2011a). However, we had to simplify a synthetic route for new methylcellulose derivatives possessing lower critical solution temperature (LCST) behaviors in aqueous solution. Glycosylation of a cellobiose derivative with a polymeric methyl tri- $O$-methylcelluloside having one hydroxy group at the C-4 position of the glucosyl residue at the non-reducing end consumed a large amount of cellobiosyl trichloroacetimidate derivative to afford only the diblock methylcellulose. To improve the efficiency of the coupling reaction between the hydrophobic and hydrophilic segments, we synthesized a diblock methylcellulose analogue via Huisgen 1,3- dipolar cycloaddition (Nakagawa, Kamitakahara, \& Takano, 2012). A 2-propynyl group was introduced to the $\mathrm{C}-4$ hydroxy group at the non-reducing end of the methyl tri- $O$-methylcelluloside. Huisgen 1,3-dipolar cycloaddition was more efficient than glycosylation for connecting the hydrophobic and hydrophilic segments.

Recently, we have reported a versatile pathway to heterobifunctional/telechelic cellulose ethers, such as tri- $O$-methylcellulosyl azide and propargyl tri- $O$-methylcelluloside, with one free $\mathrm{C}-4$ hydroxy group attached to the glucosyl residue at the non-reducing end for the use in the Huisgen 1,3-dipolar cycloaddition (Hiroshi Kamitakahara et al., 2016). This new method enables us to prepare a hydrophobic segment for the Huisgen 1,3-dipolar cycloaddition from 
65

66

67

tri-O-methylcellulose in a one-step reaction.

If the chemical structure of trehalose-type diblock polysaccharide analogues exhibited the same physical properties as those of the original diblock polysaccharides, the Huisgen 1,3-dipolar cycloaddition of azido and alkyne derivatives could produce a variety of diblock polysaccharide analogues more easily than a glycosylation method, to afford, for instance,

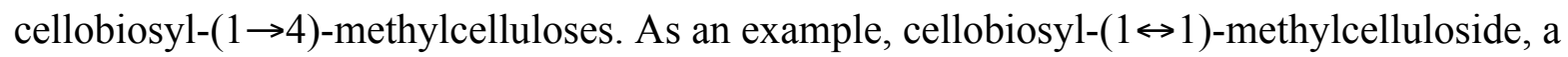
trehalose-type diblock copolymer, possesses an analogous structure to cellobiosyl-( $1 \rightarrow 4)$-methylcellulose. Moreover, 1-methylcellulosyl-4-cellobiosyloxymethyl-1H-1,2,3-triazole and 4-methylcellulosyloxymethyl-1-cellobiosyl-1 $H$-1,2,3-triazole have analogous structures to cellobiosyl-( $1 \leftrightarrow 1)$-methylcelluloside. Therefore, 1-methylcellulosyl-4-cellobiosyloxymethyl-1H-1,2,3-triazole and 4-methylcellulosyloxymethyl-1-cellobiosyl-1H-1,2,3-triazole exhibit analogous structures to cellobiosyl-(1 $\rightarrow 4)$-methylcellulose, a diblock methylcellulose. These triazole-linked diblock methylcellulose analogues would allow us to gain deep insights into not only fundamental but also potential properties of methylcelluloses.

A hydrophilic segment would be chosen to tune the properties of the methylcellulose, thereby producing new functional methylcellulose derivatives. Methylcellulose is nonionic. Cationic and anionic cellulose ethers are also of industrial importance. Commercial cationic hydroxyethyl cellulose (QC-10), O-[2-hydroxy-3-(trimethylammonio)]propyl hydroxyethyl cellulose chloride, is well known as a conditioning polymer for hair-care products (Hossel, Dieing, Norenberg, Pfau, \& Sander, 2000). Chitosan, poly(2-amino-2-deoxy-glucopyranose), an analogous structure to cellulose, is the second most abundant natural polymer (Rinaudo, 2006). 6-Amino-6-deoxycellulose (Teshirogi, Yamamoto, Sakamoto, \& Tonami, 1979) is an analogous polymer to chitosan. Carboxymethyl cellulose (Heinze, Erler, Nehls, \& Klemm, 1994) is an anionic cellulose ether, and its application fields are widely spread. Recently, cellouronic acid (Isogai \& Kato, 1998) and cellulose nanofibers prepared by TEMPO (2,2,6,6-tetramethylpiperidinyloxy) oxidation (Saito, Kimura, Nishiyama, \& Isogai, 2007; Saito, Nishiyama, Putaux, Vignon, \& Isogai, 2006) have gained increasing attention as anionic cellulosic materials.

To gain deep insights into the influence of the hydrophilic segments of the diblock methylcellulose analogues on the general properties of the original methylcellulose, we chose three hydrophilic segments: $\beta$-D-glucopyranosyl-( $1 \rightarrow 4)-\beta$-D-glucopyranose, (6-amino-6-deoxy- $\beta$-D-glucopyranosyl)-(1 $\rightarrow 4)-6$-amino-6-deoxy- $\beta$-D-glucopyranose, and $(\beta$-D-glucopyranuronosyl)-( $1 \rightarrow 4)-\beta$-D-glucopyranuronic acid. 
99 Methylcellulose-based diblock copolymers bearing cationic or anionic hydrophilic segments would 100 enhance the physical performance of commercially available methylcellulose. Thus, we describe, in 101 this paper, the synthesis and structure-property relationships of

102 1-(tri- $O$-methyl-cellulosyl)-4-( $\beta$-D-glucopyranosyl-( $1 \rightarrow 4)$ - $\beta$-D-glucopyranosyloxymethyl)- $1 H$ - $1,2,3$ 103 -triazole (1),

104 1-(tri- $O$-methyl-cellulosyl)-4-((6-amino-6-deoxy- $\beta$-D-glucopyranosyl)-( $1 \rightarrow 4)-6$-amino-6-deoxy- $\beta$ 105 D-glucopyranosyloxymethyl)-1 $H$-1,2,3-triazole (2), and

106 4-(tri- $O$-methyl-cellulosyloxymethyl)-1-(( $\beta$-D-glucopyranuronosyl)-(1 $\rightarrow 4)-\beta$-D-glucopyranuronosyl 107 )-1H-1,2,3-triazole (3). In particular, their surface activities, thermal properties, and 108 thermoresponsive gelation properties will be discussed.

\section{Experimental}

\subsection{General measurements}

$111{ }^{1} \mathrm{H}$ - and ${ }^{13} \mathrm{C}-\mathrm{NMR}$ spectra were recorded with Varian $500 \mathrm{NMR}$ (500 MHz) or Varian INOVA300

$112(300 \mathrm{MHz})$ spectrometer in chloroform- $d$ with tetramethylsilane as an internal standard or in

113 deuterium oxide with 3-(trimethylsilyl)-1-propanesulfonic acid sodium salt as an external standard.

114 Chemical shifts $(\delta)$ and coupling constants $(J)$ are given in ppm and $\mathrm{Hz}$, respectively.

115 Matrix-assisted laser desorption/ionization time-of-flight mass spectrometry (MALDI-TOF MS) 116 analysis was performed with a Bruker MALDI-TOF MS Autoflex III in the positive ion and linear 117 modes. For ionization, a smartbeam laser was used. All spectra were measured in the linear mode 118 by using external calibration. MALDI-TOF MS used 2,5-dihydroxybenzoic acid as a matrix. A 119 Shimadzu liquid chromatography injector (LC-10ATvp), Shimadzu column oven (CTO-10Avp), 120 Shimadzu ultraviolet visible detector (SPD-10Avp), Shimadzu refractive index detector (RID-10A), 121 Shimadzu communication bus module (CBM-10A), Shimadzu LC workstation (CLASS-LC10), 122 and Shodex columns (KF802, KF802.5, and KF805) were used. Number- and weight-averaged 123 molecular weights $\left(M_{\mathrm{n}}, M_{\mathrm{w}}\right)$ and polydispersity indices $\left(M_{\mathrm{w}} / M_{\mathrm{n}}\right)$ were estimated by using 124 polystyrene standards (Shodex). A flow rate of $1 \mathrm{~mL} / \mathrm{min}$ at $40^{\circ} \mathrm{C}$ was chosen. Chloroform was 125 used as the eluent.

\subsection{Differential scanning calorimetry (DSC) measurements}

127 DSC thermograms were recorded on a DSC823 ${ }^{\mathrm{e}}$ instrument (Mettler Toledo, Zurich, Switzerland) with an HSS7 sensor under a nitrogen atmosphere during a heating/cooling cycle $\left(0 \rightarrow 90 \rightarrow 0{ }^{\circ} \mathrm{C}\right)$ with a heating and cooling rate of $3.5{ }^{\circ} \mathrm{C} / \mathrm{min}$. Each temperature cycle was sequentially repeated three times in order to ensure and check the reproducible response of the instrument. The sample concentration for DSC measurements was $2.0 \mathrm{wt} . \%$. 
132

133

134

135

136

137

138

139

\subsection{Dynamic light scattering (DLS) measurements}

DLS measurements were performed with an ELS-Z zeta-potential and particle-size analyzer (Otsuka Electronics Co., Ltd, Osaka, Japan) and observed in the temperature range from 10 to $90{ }^{\circ} \mathrm{C}$. The sample solutions were kept for $5 \mathrm{~min}$ at the required temperature before each measurement. The sample concentration for DLS measurements was 0.2 or $2.0 \mathrm{wt}$ \%. The hydrodynamic diameters were obtained by Cumulant method. Intensity and number size distributions were obtained by Marquardt method.

\subsection{Surface tension measurements}

Surface tension was measured by the Wilhelmy method by using a CBVP-A3 surface tensiometer (Kyowa Interface Science, Co. Ltd., Tokyo, Japan) at $25{ }^{\circ} \mathrm{C}$. A Teflon cell containing $700 \mu \mathrm{L}$ of solution was used for the measurement. The surface tension gradually decreased during the measurements. The values were stable after $30 \mathrm{~min}$ and were recorded.

\subsection{Scanning electron microscopy (SEM) and transmission electron microscopy (TEM)}

The three kinds of hydrogels from aqueous solutions of compounds $\mathbf{1}, \mathbf{2}$ and $\mathbf{3}$ were frozen with liquid nitrogen, lyophilized, and cut with a razor blade. The cut surfaces of the hydrogels were sputter-coated with gold with an ion-coater (JFC-1100E, JEOL, Tokyo, Japan) and observed under a scanning electron microscope (JSM-6060, JEOL) at an accelerating voltage of $5 \mathrm{kV}$.

A drop of aqueous dispersion of compound 1 was mounted on a copper grid with an elastic carbon supporting film (Oken Shoji, Tokyo, Japan) and observed under a transmission electron microscope (JEM1400, JEOL) at an accelerating voltage of $100 \mathrm{kV}$ after negative staining with uranyl acetate.

\subsection{Syntheses}

Cellobiose octaacetate (12):

Cellobiose was acetylated to give cellobiose octaacetate (12) according to the method in our previous paper (H. Kamitakahara, Nakatsubo, \& Klemm, 2006). Cellobiose (12.04 g, 35.17 mmol) and sodium acetate were added in acetic anhydride $(60 \mathrm{~mL})$. The reaction mixture was stirred at $55^{\circ} \mathrm{C}$ over night and $100{ }^{\circ} \mathrm{C}$ for $3 \mathrm{~h}$. The reaction mixture was poured into water with ice $(600 \mathrm{~mL})$. Crude crystals were filtered and washed with distilled water and recrystallized with EtOH to give colorless crystals. (20.8 g, 30.65 mmol, 87\% yield). CAS Registry No. 5346-90-7

2-Propynyl (2,3,6-tri- $O$-acetyl- $\beta$-D-glucopyranosyl)-( $1 \rightarrow 4)-2,3,6$-tri- $O$-acetyl- $\beta$-D-glucopyranoside (9) (Moni et al., 2013): 
164

165

166

167

168

169

170

171

172

173

174

175

176

177

178

179

180

181

182

183

184

185

186

187

188

189

190

191

192

193

194

195

196

197

198

199
2-Propyne-1-ol (1.05 mL, $18.2 \mathrm{mmol}, 1.2$ equiv.) was added to a solution of compound 12 (10.3 g, $15.2 \mathrm{mmol})$ in anhydrous dichloromethane $(40 \mathrm{~mL})$. The reaction mixture was cooled to $0{ }^{\circ} \mathrm{C}$.

Boron trifluoride diethyl ether complex $(2.86 \mathrm{~mL}, 22.8 \mathrm{mmol}, 1.5$ equiv.) was added to the reaction mixture at $0{ }^{\circ} \mathrm{C}$. The mixture was stirred for about one day. Solid $\mathrm{NaHCO}_{3}$ was then added to the reaction mixture. The reaction mixture was extracted with dichloromethane, washed with water, sat. aq. $\mathrm{NaHCO}_{3}$ solution, and brine, dried with $\mathrm{Na}_{2} \mathrm{SO}_{4}$, and concentrated to dryness. The obtained crude crystals were recrystallized with dichloromethane/n-hexane to produce 2-propynyl $(2,3,6$-tri- $O$-acetyl- $\beta$-D-glucopyranosyl)-( $1 \rightarrow 4)$-2,3,6-tri- $O$-acetyl- $\beta$-D-glucopyranoside $(\mathbf{9}, 10.1223$ g, 99\% yield).

${ }^{1} \mathrm{H}$ NMR (Moni et al., 2013) (400 MHz): $\delta 5.21(\mathrm{dd}, 1 \mathrm{H}, J 2,3=J 3,4=9.2 \mathrm{~Hz}, \mathrm{H}-3), 5.14\left(\mathrm{dd}, 1 \mathrm{H}, J 2^{\prime}, 3^{\prime}=J 3^{\prime}, 4^{\prime}=\right.$ $\left.9.1 \mathrm{~Hz}, \mathrm{H}-3^{\prime}\right), 5.06$ (dd, 1H, H-4'), 4.93 (2 dd, 2H, H-2, H-2') 4.73 (d, 1H, J1,2 = 8.0 Hz, H-1), 4.54 (dd, 1H, J5,6a = 2.0 $\mathrm{Hz}, J 6 \mathrm{a}, 6 \mathrm{~b}=12.0 \mathrm{~Hz}, \mathrm{H6a}), 4.51$ (d, 1H, J1',2' = 8.0 Hz, H-1'), 4.37 (dd, 1H, J5',6'a = 4.5 Hz, J6'a, 6'b = 12.5 Hz, H6'a), $4.33\left(\mathrm{~d}, 2 \mathrm{H}, J=2.5 \mathrm{~Hz}, \mathrm{OCH}_{2} \mathrm{CCH}\right), 4.10(\mathrm{dd}, 1 \mathrm{H}, J 5,6 \mathrm{~b}=4.7 \mathrm{~Hz}, \mathrm{H}-6 \mathrm{~b}), 4.14$ (dd, 1H, J5',6'b = 2.0 Hz, H-6'b), 3.79 (dd, 1H, H-4), 3.68-3.60 (m, 2H, H-5, H-5' ), 2.45 (t, 1H, $\left.\mathrm{OCH}_{2} \mathrm{CCH}\right), 2.19-2.01$ (7 s, 21H, $7 \mathrm{Ac}$ ).

${ }^{13} \mathrm{C}-\mathrm{NMR}\left(125 \mathrm{MHz}, \mathrm{CDCl}_{3}\right)$ : $\delta$ 20.5, 20.6, 20.7, 20.8, 55.9 (- $\left.\mathrm{CH}_{2} \mathrm{CCH}\right), 61.5\left(\mathrm{C}^{\prime}\right), 61.7(\mathrm{C} 6), 67.8$ (C4'), 71.2 (C2), 71.6 (C2'), 71.9 (C5'), 72.4 (C3), 72.8 (C5 or C3'), 72.9 (C5 or C3'), 75.4 $\left(\mathrm{CH}_{2} \mathrm{C} \underline{\mathrm{CH}}\right), 76.3(\mathrm{C} 4), 78.0\left(\mathrm{CH}_{2} \underline{\mathrm{CCH}}\right), 97.9$ (C1), 100.7 (C1'), 169.0, 169.3, 169.7, 169.7, 170.2, $170.3,170.5$

2-Propynyl $\beta$-D-cellobioside (13) (Moni et al., 2013):

Sodium methoxide (28\%) in methanol $(0.48 \mathrm{~mL}, 8.37 \mathrm{mmol}, 1.4$ equiv.) was added to a solution of compound 9 (4.0010 g, $5.93 \mathrm{mmol})$ in tetrahydrofuran (THF; $100 \mathrm{~mL})$ and methanol $(50 \mathrm{~mL})$. The reaction mixture was stirred for $3.3 \mathrm{~h}$ at room temperature. Amberlyst $\mathrm{H}^{+}$was added to neutralize the mixture and was then filtered off. The combined filtrate and washings were then concentrated to dryness to give 2-propynyl $\beta$-D-cellobioside $(13,2.17 \mathrm{~g}, 96 \%$ yield $)$.

${ }^{1} \mathrm{H}$ NMR (400 MHz, D $2 \mathrm{O}$ ) (Moni et al., 2013): $\delta 4.50$ (d, 1H, $J 1,2=8.0 \mathrm{~Hz}, \mathrm{H}-1$ ), 4.34 (d, 1H, $J 1^{\prime}, 2^{\prime}=7.5 \mathrm{~Hz}$, H-1' ), 4.31 (dd, $\left.2 \mathrm{H}, J=16.0,2.5 \mathrm{~Hz}, \mathrm{OCH}_{2} \mathrm{CCH}\right), 3.82(\mathrm{dd}, 1 \mathrm{H}, J 5,6 \mathrm{a}=2.0 \mathrm{~Hz}, J 6 \mathrm{a}, 6 \mathrm{~b}=12.5 \mathrm{~Hz}, \mathrm{H}-6 \mathrm{a}), 3.75(\mathrm{dd}, 1 \mathrm{H}$, $\left.J 5^{\prime}, 6{ }^{\prime} \mathrm{a}=2.0 \mathrm{~Hz}, J 6^{\prime} \mathrm{a}, 6^{\prime} \mathrm{b}=12.2 \mathrm{~Hz}, \mathrm{H6} 6^{\prime} \mathrm{a}\right), 3.65(\mathrm{dd}, 1 \mathrm{H}, J 5,6 \mathrm{~b}=4.5 \mathrm{~Hz}, \mathrm{H}-6 \mathrm{~b}), 3.56$ (dd, 1H, J5',6'b = 5.5 Hz, H-6b'), 3.51-3.42 (m, 3H, H-3, H-4, H-5), 3.36-3.12 (m, 5H, H-2, H-2' , H-3' , H-4' , H-5' ), 2.75 (t, 1H, OCH $2 \mathrm{CCH}$ ).

${ }^{13} \mathrm{C}-\mathrm{NMR}\left(125 \mathrm{MHz}, \mathrm{D}_{2} \mathrm{O}\right): \delta 51.5,59.3,62.6,63.2,72.1,75.4,75.8,76.9,77.5,78.2,78.7,79.0$, 81.2, 81.4, 103.0, 105.2

\section{2-Propynyl}

(6-O- $p$-toluenesulfonyl- $\beta$-D-glucopyranosyl)-( $1 \rightarrow 4)$-6- $O$ - $p$-toluenesulfony- $\beta$-D-glucopyranoside (14):

Tosyl chloride ( $3.48 \mathrm{~g}, 18.3 \mathrm{mmol}$ ) was added at $0{ }^{\circ} \mathrm{C}$ to a solution of 2-propynyl $\beta$-D-cellobioside 
$200(\mathbf{1 3}, 2.17 \mathrm{~g}, 5.69 \mathrm{mmol})$ in pyridine $(8 \mathrm{~mL})$. The reaction mixture was stirred at $8{ }^{\circ} \mathrm{C}$ for $22.5 \mathrm{~h}$.

201 Brine was then added to the reaction mixture. The organic phase was extracted with ethyl acetate 202 three times, and pyridine was azeotropically removed with ethanol to produce crude compound 14.

203 The crude product was purified by silica gel column chromatography (methanol/chloroform=1/5, $204 \mathrm{v} / \mathrm{v}$ ) to give 2-propynyl

205 (6-O-p-toluenesulfonyl- $\beta$-D-glucopyranosyl)-(1 $\rightarrow 4)-6-O-p$-toluenesulfonyl- $\beta$-D-glucopyranoside $206 \quad(14,1.97 \mathrm{~g}, 50.5 \%$ yield $)$.

$207{ }^{1} \mathrm{H}-\mathrm{NMR}\left(500 \mathrm{MHz}, \mathrm{CDCl}_{3}\right): \delta 2.40\left(\mathrm{~s}, 3 \mathrm{H}, \mathrm{PhCH}_{3}\right), 2.41\left(\mathrm{~s}, 3 \mathrm{H}, \mathrm{PhCH}_{3}\right), 2.49\left(\mathrm{t}, 1 \mathrm{H},-\mathrm{CH}_{2} \mathrm{CCH}\right)$, 2083.36 (t, $1 \mathrm{H}, J=8.5 \mathrm{~Hz}), 3.45$ (t, $1 \mathrm{H}, J=8.5 \mathrm{~Hz}), 3.49-3.70$ (m), 4.17 (dd, 1H, $J=2 \mathrm{~Hz}, J=16 \mathrm{~Hz}$, $209 \mathrm{CH} \mathrm{CHCH}_{2}, 4.24-4.43$ (H6', H6', H6, H6, $\left.\mathrm{CH}_{2} \mathrm{CCH}\right), 4.44$ (d, 1H, J=8.0 Hz, H1), 4.50 (d, 1H, J=7.0 $\left.210 \mathrm{~Hz}, \mathrm{H} 1^{\prime}\right)$, 7.3-7.8 (aromatic $\mathrm{H}$ )

$211{ }^{13} \mathrm{C}-\mathrm{NMR}\left(125 \mathrm{MHz}, \mathrm{CDCl}_{3}\right): \delta$ 21.7, $55.9\left(-\mathrm{CH}_{2} \mathrm{CCH}\right), 68.9$ (C6'), 69.1 (C6), 69.4 (C3'), 72.1, $21272.4,73.0(\mathrm{C} 2), 73.5,73.7,75.7,75.7\left(\mathrm{CH}_{2} \mathrm{CCH}\right), 77.0,78.8\left(\mathrm{CH}_{2} \underline{\mathrm{CCH}}\right)$, 100.0, $101.3\left(\mathrm{C} 1^{\prime}\right)$, 128.0, $213129.8,130.0,132.2,132.6,144.9,145.2$ (aromatic C)

2-Propynyl

216 (2,3,4-tri- $O$-acetyl-6- $O$ - $p$-toluenesulfonyl- $\beta$-D-glucopyranosyl)-(1 $\rightarrow 4)$-2,3-di- $O$-acetyl-6- $O$ - $p$-tolue 217 nesulfonyl- $\beta$-D-glucopyranoside (15):

218 Acetic anhydride $(1 \mathrm{~mL})$ was added to a solution of 2-propynyl

219 (6-O-p-toluenesulfonyl- $\beta$-D-glucopyranosyl)-(1 $\rightarrow 4)-6-O$ - $p$-toluenesulfonyl- $\beta$-D-glucopyranoside $220(\mathbf{1 4}, 0.763 \mathrm{~g})$ in pyridine $(5 \mathrm{~mL})$. The reaction mixture was stirred at room temperature overnight. 221 The reaction mixture was extracted with ethyl acetate, washed with $1 \mathrm{~N} \mathrm{HCl}$, sat. aq. $\mathrm{NaHCO}_{3}$, and 222 brine, dried over $\mathrm{Na}_{2} \mathrm{SO}_{4}$, and concentrated to dryness to give 2-propynyl

223 (2,3,4-tri- $O$-acetyl-6- $O$ - $p$-toluenesulfonyl- $\beta$-D-glucopyranosyl)-(1 $\rightarrow 4)$-2,3-di- $O$-acetyl-6- $O$ - $p$-tolue 224 nesulfonyl- $\beta$-D-glucopyranoside $(15,0.9409 \mathrm{~g}, 94.6 \%$ yield $)$.

$225{ }^{1} \mathrm{H}-\mathrm{NMR}\left(300 \mathrm{MHz}, \mathrm{CDCl}_{3}\right): \delta 1.91,1.98,1.99,2.00,2.03\left(\mathrm{COCH}_{3}\right), 2.45\left(\mathrm{t}, 1 \mathrm{H},-\mathrm{CH}_{2} \mathrm{CCH}\right)$, $2262.47\left(\mathrm{~s}, 3 \mathrm{H}, \mathrm{PhCH}_{3}\right), 2.48\left(\mathrm{~s}, 3 \mathrm{H}, \mathrm{PhCH}_{3}\right), 3.55(\mathrm{~m}, 1 \mathrm{H}, J=2.0, J=3.5 \mathrm{~Hz}, J=9.5 \mathrm{~Hz}, \mathrm{H} 5), 3.65$ (m, $2271 \mathrm{H}, J=2.5 \mathrm{~Hz}, J=4.5 \mathrm{~Hz}, J=10.0 \mathrm{~Hz}, \mathrm{H} 5$ '), 3.71 (t, 1H, $J=10.0 \mathrm{~Hz}, \mathrm{H} 4), 4.10$ (dd, $1 \mathrm{H}, J=5.0 \mathrm{~Hz}$, $228 J=11.5$ Hz, H6'), 4.14-4.28 (H6', H6, CH $\mathrm{CCH}$ ), 4.33 (dd, 1H, J=2.0 Hz, J=11.0 Hz, H6), 4.38 (d, $\left.2291 \mathrm{H}, J=7.5 \mathrm{~Hz}, \mathrm{H} 1^{\prime}\right), 4.65$ (d, $1 \mathrm{H}, J=8.5 \mathrm{~Hz}, \mathrm{H} 1$ ), 4.80 (t, 1H, $\left.J=9.5 \mathrm{~Hz}, \mathrm{H} 2\right), 4.81$ (t, $1 \mathrm{H}, J=8.5 \mathrm{~Hz}$, $230 \mathrm{H} 2$ '), 4.93 (t, 1H, J=10.0 Hz, H4'), 5.03 (t, 1H, J=9.0 Hz, H3'), 5.11 (t, 1H, J=9.0 Hz, H3), 231 7.39-7.84 (aromatic $\mathrm{H})$

$232{ }^{13} \mathrm{C}-\mathrm{NMR}\left(75 \mathrm{MHz}, \mathrm{CDCl}_{3}\right): \delta$ 20.5, 20.5, 20.6, 20.7, 21.6, 21.7, $55.7\left(-\underline{C H}_{2} \mathrm{CCH}\right), 66.3(\mathrm{C} 6$ '), 66.8 233 (C6), 68.0 (C4'), 70.8 (C2), 71.4 (C2'), 71.5 (C5'), 71.8 (C3), 72.4 (C5), 72.8 (C3'), 74.9 (C4), 75.6 $234\left(\mathrm{CH}_{2} \mathrm{CCH}\right), 77.9\left(\mathrm{CH}_{2} \underline{\mathrm{CCH}}\right), 97.7(\mathrm{C} 1), 100.0\left(\mathrm{C} 1^{\prime}\right), 128.0,128.1,130.1,130.1,132.3,132.6$, $235145.4,145.5$ (aromatic C), 168.8, 169.3, 169.5, 169.9, $170.1\left(\mathrm{COCH}_{3}\right)$ 
2-Propynyl

(2,3,4-tri- $O$-acetyl-6-azido-6-deoxy- $\beta$-D-glucopyranosyl)-( $1 \rightarrow 4)$-2,3-di- $O$-acetyl-6-azido-6-deoxy- $\beta$

-D-glucopyranoside (16):

Sodium azide $(0.3731 \mathrm{~g})$ was added to a solution of 2-propynyl

(2,3,4-tri- $O$-acetyl-6- $O$ - $p$-toluenesulfonyl- $\beta$-D-glucopyranosyl)-( $1 \rightarrow 4)$-2,3-di- $O$-acetyl-6- $O$ - $p$-tolue nesulfonyl- $\beta$-D-glucopyranoside $(\mathbf{1 5}, 1.2888 \mathrm{~g}$ ) in $N, N$-dimethylformamide (DMF; $5 \mathrm{~mL}$ ). The reaction mixture was stirred overnight at $50{ }^{\circ} \mathrm{C}$. The mixture was then poured into distilled water with ice. The organic phase was extracted with dichloromethane four times, dried over sodium sulfate, and concentrated to dryness. The crude product was purified by silica gel column chromatography (eluent: ethyl acetate $/ n$-hexane $=2 / 1, \mathrm{v} / \mathrm{v}$ ) to afford 2-propynyl $(2,3,4$-tri- $O$-acetyl-6- $O$-azido-6- $O$-deoxy- $\beta$-D-glucopyranosyl)-( $1 \rightarrow 4)-2,3$-di- $O$-acetyl-6- $O$-azido-6$O$-deoxy- $\beta$-D-glucopyranoside (16, $0.8904 \mathrm{~g}, 97 \%$ yield).

${ }^{1} \mathrm{H}-\mathrm{NMR}\left(500 \mathrm{MHz}, \mathrm{CDCl}_{3}\right): \delta 1.99\left(\mathrm{~s}, 3 \mathrm{H},(\mathrm{CO}) \mathrm{CH}_{3}\right), 2.04\left(\mathrm{~s}, 3 \mathrm{H},(\mathrm{CO}) \mathrm{CH}_{3}\right), 2.05(\mathrm{~s}, 3 \mathrm{H}$, $\left.(\mathrm{CO}) \mathrm{CH}_{3}\right), 2.05\left(\mathrm{~s}, 3 \mathrm{H},(\mathrm{CO}) \mathrm{CH}_{3}\right), 2.08\left(\mathrm{~s}, 3 \mathrm{H},(\mathrm{CO}) \mathrm{CH}_{3}\right), 2.47$ (s, $\left.1 \mathrm{H}, J=2.5 \mathrm{~Hz}, \mathrm{CH}_{2} \mathrm{CC} \underline{\mathrm{H}}\right), 3.37$ (dd, 1H, $J=5.0 \mathrm{~Hz}, J=13.0 \mathrm{~Hz}, \mathrm{H6}$ ') 3.40 (dd, 1H, $J=4.5 \mathrm{~Hz}, J=13.0 \mathrm{~Hz}, \mathrm{H} 6$ ), 3.43 (dd, 1H, $J=3.0$ Hz, $J=13.5$ Hz, H6'), 3.58 (dd, 1H, $J=2.0$ Hz, J=13.0 Hz, H6), 3.60-3.64 (2H, m, H5, H5'), 3.85 (t, $1 \mathrm{H}, J=9.5 \mathrm{~Hz}, \mathrm{H} 4), 4.36$ (d, 2H, $J=2.5 \mathrm{~Hz}, \mathrm{CH}_{2} \mathrm{CCH}$ ), 4.57 (d, $\left.1 \mathrm{H}, J_{1^{\prime}, 2},=8.0 \mathrm{~Hz}, \mathrm{H} 1^{\prime}\right), 4.79$ (d, $1 \mathrm{H}$, $J_{1,2}=8.0 \mathrm{~Hz}, \mathrm{H} 1$ ), 4.89 (dd, 1H, $J=8.0 \mathrm{~Hz}, J=9.5 \mathrm{~Hz}, \mathrm{H} 2$ '), 4.94 (dd, 1H, $J=8.0 \mathrm{~Hz}, J=9.5 \mathrm{~Hz}, \mathrm{H} 2$,), 4.99 (t, 1H, J=9.5 Hz, H4'), 5.16 (t, 1H, $J=9.5$ Hz, H3'), 5.22 (t, 1H, J=9.5 Hz, H3)

${ }^{13} \mathrm{C}-\mathrm{NMR}\left(125 \mathrm{MHz}, \mathrm{CDCl}_{3}\right): \delta$ 20.5, 20.6, 20.6, 20.7, $20.8\left((\mathrm{CO}) \mathrm{CH}_{3}\right), 50.2(\mathrm{C} 6), 50.9\left(\mathrm{C}^{\prime}\right), 55.8$ $\left(\mathrm{CH}_{2} \mathrm{CCH}\right), 69.1$ (C4'), 71.3 (C2), 71.7 (C2'), 72.4 (C3), 72.6 (C3'), 72.7 (C5), 74.5 (C5'), 75.6 $\left(\mathrm{CH}_{2} \mathrm{CCH}\right), 75.9(\mathrm{C} 4), 78.0\left(\mathrm{CH}_{2} \underline{\mathrm{CCH}}\right), 97.8(\mathrm{C} 1), 100.1\left(\mathrm{C} 1^{\prime}\right), 168.9,169.4,169.6,169.8,170.2$ $\left((\mathrm{CO}) \mathrm{CH}_{3}\right)$

2-Propynyl (6-azido-6-deoxy- $\beta$-D-glucopyranosyl)-( $1 \rightarrow 4)$-6-azido-6-deoxy- $\beta$-D-glucopyranoside 263 (17):

Sodium methoxide $(28 \%)$ in methanol $(28 \mu \mathrm{L})$ was added to 2-propynyl

(2,3,4-tri- $O$-acetyl-6-azido-6-deoxy- $\beta$-D-glucopyranosyl)-(1 $\rightarrow 4)$-2,3-di- $O$-acetyl-6-azido-6-deoxy- $\beta$ -D-glucopyranoside $(\mathbf{1 6}, 0.3028 \mathrm{~g})$ in methanol $(1.5 \mathrm{~mL})$ and THF $(1.5 \mathrm{~mL})$. The reaction mixture was stirred at room temperature for overnight. The mixture was neutralized with Amberlyst $\mathrm{H}^{+}$. The Amberlyst $\mathrm{H}^{+}$was removed by filtration and washed with methanol. The filtrate and washings were concentrated to dryness to produce 2-propynyl 
272

273

274

275

276

277

278

279

280

281

282

283

284

285

286

287

288

289

290

291

292

293

294

295

296

297

298

299

300

301

302

303

304

305

306

307

${ }^{1} \mathrm{H}-\mathrm{NMR}\left(500 \mathrm{MHz}, \mathrm{D}_{2} \mathrm{O}\right): \delta 2.92\left(\mathrm{t}, 1 \mathrm{H}, J=2.5 \mathrm{~Hz}, \mathrm{CH}_{2} \mathrm{CC} \underline{H}\right.$ ), 3.30 (dd, $1 \mathrm{H}, J=8.0 \mathrm{~Hz}, J=9.0 \mathrm{~Hz}$, H2'), 3.35 (dd, 1H, J=8.0 Hz, J=9.5 Hz, H2), 3.43 (t, 1H, J=9.0 Hz, H4'), 3.48 (t, 1H, J=9.0 Hz, H3'), 3.52 (dd, 1H, $J=5.5 \mathrm{~Hz}, J=13.0 \mathrm{~Hz}, \mathrm{H6}$ '), 3.58 (m, 1H, $J=9.25 \mathrm{~Hz}, J=2.3 \mathrm{~Hz}, 5.5 \mathrm{~Hz}, \mathrm{H} 5$ '), 3.61-3.66 (3H, m, H3, H4, H6), 3.75 (dd, 1H, $J=2.5$ Hz, $J=14.0$ Hz, H6), 3.75-3.79 (1H, m, H5), 3.78 (dd, 1H, $J=2.5 \mathrm{~Hz}, J=13.5 \mathrm{~Hz}, \mathrm{H} 6$ ') $4.46\left(2 \mathrm{H},\left(\mathrm{C}_{2} \underline{\mathrm{CCH}}\right)\right), 4.49$ (d, 1H, $\left.J=8 \mathrm{~Hz}, \mathrm{H} 1^{\prime}\right), 4.68$ (d, 1H, J=7.5 Hz, H1)

${ }^{13} \mathrm{C}-\mathrm{NMR}\left(\mathrm{D}_{2} \mathrm{O}\right): \delta 50.2$ (C6 (b)), 50.8 (C6 (a)), $56.7\left(\mathrm{CH}_{2} \mathrm{CCH}\right), 70.0(\mathrm{C} 4$ ') $, 72.6(\mathrm{C} 2), 73.0(\mathrm{C} 2$ '), 73.6 (C5), 73.9 (C3), 74.2 (C5'), 75.2 (C3'), $76.4\left(\mathrm{CH}_{2} \mathrm{CC} H\right), 78.5\left(\mathrm{CH}_{2} \underline{\mathrm{CCH}}\right), 79.1$ (C4), 100.4 (C1), 102.5 (C1')

$\mathrm{Mw}=430.4$ MALDI-TOF MS: $m / z[\mathrm{M}+\mathrm{Na}]^{+}=453.1, \mathrm{~m} / z[\mathrm{M}+\mathrm{K}]^{+}=469.1$

2-Propynyl (6-amino-6-deoxy- $\beta$-D-glucopyranosyl)-( $1 \rightarrow 4)$-6-amino-6-deoxy- $\beta$-D-glucopyranoside (18):

Triphenylphosphine (132.1 mg) was added to a solution of 2-propynyl

(6-azido-6-deoxy- $\beta$-D-glucopyranosyl)-(1 $\rightarrow 4$ )-6-azido-6-deoxy- $\beta$-D-glucopyranoside $(17,54.2 \mathrm{mg})$ in methanol $(3 \mathrm{~mL})$, THF $(3 \mathrm{~mL})$, and distilled water $(0.7 \mathrm{~mL})$. The reaction mixture was stirred at room temperature for 14 days. The reaction product was extracted with distilled water and washed with dichloromethane three times. The water layer was concentrated to dryness to afford 2-propynyl (6-amino-6-deoxy- $\beta$-D-glucopyranosyl)-( $1 \rightarrow 4)$-6-amino-6-deoxy- $\beta$-D-glucopyranoside $(\mathbf{1 8}, 46.8$ $\mathrm{mg}, 98 \%$ yield).

${ }^{1} \mathrm{H}-\mathrm{NMR}\left(\mathrm{D}_{2} \mathrm{O}\right): \delta 2.79$ (dd, $\left.1 \mathrm{H}, J=7.5 \mathrm{~Hz}, J=13.5 \mathrm{~Hz}, \mathrm{H} 6\right), 2.80$ (dd, 1H, $J=7.5 \mathrm{~Hz}, J=14.0 \mathrm{~Hz}, \mathrm{H} 6$ '), 3.07 (dd, 1H, $J=3.0$ Hz, $J=14.0$ Hz, H6'), 3.19 (dd, 1H, $J=2.0$ v, $J=13.0$ Hz, H6), 3.29 (dd, 1H, $J=7.5$ Hz, $J=9.0$ Hz, H2'), 3.31 (t, 1H, J=8.0 Hz, H4') 3.33 (t, 1H, J=8.0 Hz, H2), 3.39 (m, 1H, J=9.5 Hz, $J=2.5 \mathrm{~Hz}, J=7.0 \mathrm{~Hz}, \mathrm{H} 5$ '), 3.47 (t, 1H, $J=9.5 \mathrm{~Hz}, \mathrm{H} 3$ '), 3.51-3.561 (m, 2H, H4, H5), 3.60 (t, 1H, $J=8.5 \mathrm{~Hz}, \mathrm{H} 3$ ), 4.46 (d, 2H, $\left.J=1.5 \mathrm{~Hz}, \underline{\mathrm{CH}}_{2} \mathrm{CCH}\right), 4.47$ (d, 1H, J=8.0 Hz, H1'), 4.64 (d, 1H, $J=8.0$ $\mathrm{Hz}, \mathrm{H1})$

${ }^{13} \mathrm{C}-\mathrm{NMR}\left(\mathrm{D}_{2} \mathrm{O}\right): \delta 41.1$ (C6), 41.2 (C6'), $56.8\left(\mathrm{C}_{2} \mathrm{CCH}\right), 70.9(\mathrm{C} 4$ '), $72.8(\mathrm{C} 2), 73.3$ (C2'), 74.2 (C3), 75.2 (C5), $75.4\left(\mathrm{C}^{\prime}\right.$ '), 75.6 (C5'), $75.9\left(\mathrm{CH}_{2} \mathrm{CCH}\right), 78.4\left(\mathrm{CH}_{2} \underline{\mathrm{CCH}}\right), 80.2(\mathrm{C} 4), 100.8(\mathrm{C} 1)$, $102.7\left(\mathrm{C} 1^{\prime}\right)$

$\mathrm{Mw}=378.4$ MALDI-TOF MS: $m / z[\mathrm{M}+\mathrm{H}]^{+}=379.3, \mathrm{~m} / z[\mathrm{M}+\mathrm{Na}]^{+}=401.3, \mathrm{~m} / z[\mathrm{M}+\mathrm{K}]^{+}=417.2$

Note that the carbon and proton resonances of the alkyne group did not appear with a good signal-to-noise ratio, although the molecular ion peak was properly detected by MALDI-TOF MS analysis. Moreover, the NMR spectra of compounds $\mathbf{1 9}$ and $\mathbf{1 0}$ indicate that the propargyl group is not affected by the Staudinger reaction of compound $\mathbf{1 7}$ to produce compound $\mathbf{1 8 .}$ 
308

309

310

311

312

313

314

315

316

2,3,4-tri-O-acetyl-6-deoxy-6-acetylamino- $\beta$-D-glucopyranosyl-( $1 \rightarrow 4)-2,3-$ di- $O$-acetyl-6-deoxy-6-ac etylamino- $\beta$-D-glucopyranoside (19):

Sodium acetate $(34.0 \mathrm{mg})$ was added to a dispersion of 2-propynyl

(6-O-amino-6-deoxy- $\beta$-D-glucopyranosyl)-( $1 \rightarrow 4)$-6- $O$-amino-6- $O$-deoxy- $\beta$-D-glucopyranoside (18, $157.1 \mathrm{mg})$ in acetic anhydride $(3 \mathrm{~mL})$. The reaction mixture was stirred at $80^{\circ} \mathrm{C}$ for $3 \mathrm{~h}$. The mixture was extracted with ethyl acetate, washed with distilled water and brine, dried over anhydrous sodium sulfate, and concentrated to dryness. The crude product was again acetylated with acetic anhydride $(0.5 \mathrm{~mL})$ in pyridine $(2 \mathrm{~mL})$ at $80^{\circ} \mathrm{C}$ for $1 \mathrm{~h}$. The reagents were azeotropically removed with toluene to afford 2-propynyl

2,3,4-tri- $O$-acetyl-6-deoxy-6-acetylamino- $\beta$-D-glucopyranosyl-( $1 \rightarrow 4)-2,3$-di- $O$-acetyl-6-deoxy-6-ac etylamino- $\beta$-D-glucopyranoside (19, $233.4 \mathrm{mg}, 96 \%$ yield).

${ }^{1} \mathrm{H}-\mathrm{NMR}\left(\mathrm{CDCl}_{3}\right): \delta 1.98,2.03,2.04,2.05,2.07,20.8\left(21 \mathrm{H}, \mathrm{O}(\mathrm{CO}) \mathrm{CH}_{3}, \mathrm{NH}(\mathrm{CO}) \mathrm{CH}_{3}\right), 2.50(\mathrm{t}, 1 \mathrm{H}$, $J=2.0, \mathrm{CH}_{2} \mathrm{CC} \underline{\mathrm{H}}$ ), 3.40 (dd, 1H, J=6.0 Hz, $14.0 \mathrm{~Hz}, \mathrm{H6}$ ), 3.43 (dd, 1H, J=6.5 Hz, $14.5 \mathrm{~Hz}, \mathrm{H6}$ ), 3.49 (ddd, 1H, $J=3.0 \mathrm{~Hz}, J=6.0 \mathrm{~Hz}, J=14.5 \mathrm{~Hz}, \mathrm{H6}$ '), 3.58-3.62 (m, 2H, H5, H5'), 3.78 (t, 1H, $J=9.5$, H4), 3.81 (ddd, 1H, $J=3.5 \mathrm{~Hz}, J=6.0 \mathrm{~Hz}, J=14.5 \mathrm{~Hz}, \mathrm{H} 6$ ), 4.33 (dd, $1 \mathrm{H}, J=2.5 \mathrm{~Hz}, J=16 \mathrm{~Hz}$, $\left.\mathrm{C}_{2} \mathrm{CCH}\right), 4.38\left(\mathrm{dd}, 1 \mathrm{H}, J=2.5 \mathrm{~Hz}, J=16 \mathrm{~Hz}, \underline{\mathrm{C}}_{2} \mathrm{CCH}\right), 4.71(\mathrm{~d}, 1 \mathrm{H}, J=8.0 \mathrm{~Hz}, \mathrm{H} 1), 4.78$ (d, 1H, $J=7.5 \mathrm{~Hz}, \mathrm{H} 1$ '), 4.91 (t, 1H, $J=9.5 \mathrm{~Hz}, \mathrm{H} 4$ '), 4.94 (dd, 1H, $J=7.5 \mathrm{~Hz}, J=9.0 \mathrm{~Hz}, \mathrm{H} 2$ '), 4.98 (dd, 1H, $J=8.0 \mathrm{~Hz}, J=9.0 \mathrm{~Hz}, \mathrm{H} 2), 5.13$ (t, 1H, $J=9.5 \mathrm{~Hz}, \mathrm{H} 3), 5.18$ (t, 1H, $J=9.5 \mathrm{~Hz}, \mathrm{H} 3$ '), 5.97 (t, 1H, $J=6.0$ $\left.\mathrm{Hz}, \mathrm{C} 6 \mathrm{~N} \underline{H}(\mathrm{CO}) \mathrm{CH}_{3}\right), 6.63\left(\mathrm{t}, 1 \mathrm{H}, J=6.0 \mathrm{~Hz}, \mathrm{C}^{\prime} \mathrm{N} \underline{H}(\mathrm{CO}) \mathrm{CH}_{3}\right)$

${ }^{13} \mathrm{C}-\mathrm{NMR}\left(\mathrm{CDCl}_{3}\right)$ : $\delta$ 20.6, 20.7, 20.7, $20.9\left(\mathrm{CH}_{3}(\mathrm{OAc})\right), 23.0,23.3\left(\mathrm{CH}_{3}(\mathrm{NHAc})\right), 39.1$ (C6'), 39.8 (C6), 56.4 ( $\left.\underline{\mathrm{CH}}_{2} \mathrm{CCH}\right), 68.7$ (C4'), 71.4 (C2), 71.7 (C2'), 72.3 (C5'), 72.7 (C3), 72.8 (C3'), 73.5 (C5), $75.5\left(\mathrm{CH}_{2} \underline{\mathrm{CCH}}\right), 76.2(\mathrm{C} 4), 78.3\left(\mathrm{CH}_{2} \mathrm{CC} \mathrm{H}\right), 98.5(\mathrm{C} 1), 98.7$ (C1'), 169.4, 169.7, 169.8, $170.1,170.2,170.3,170.6(\mathrm{C}=\mathrm{O}(\mathrm{OAc}, \mathrm{NHAc}))$

$\mathrm{Mw}=672.6 \mathrm{MALDI}-\mathrm{TOF} \mathrm{MS}: \mathrm{m} / z[\mathrm{M}+\mathrm{Na}]^{+}=695.2, \mathrm{~m} / z[\mathrm{M}+\mathrm{K}]^{+}=711.2$

\section{2-Propynyl}

[2,3,4-tri-O-acetyl-6-(tert-butoxycarbonyl)amino-6-deoxy- $\beta$-D-glucopyranosyl]-( $1 \rightarrow 4)$-2,3-di- $O$-ac etyl-6-(tert-butoxycarbonyl)amino-6-deoxy- $\beta$-D-glucopyranoside (10):

4-Dimethylaminopyridine (DMAP; $2.7 \mathrm{mg})$ and di-tert-butyl dicarbonate $\left(\mathrm{Boc}_{2} \mathrm{O} ; 0.1 \mathrm{~mL}\right)$ were added to a solution of 2-propynyl

[2,3,4-tri- $O$-acetyl-6- $O$-(acetylamino)-6-deoxy- $\beta$-D-glucopyranosyl]-(1 $\rightarrow 4)-2,3$-di- $O$-acetyl-6- $O$-(a cetylamino)-6-deoxy- $\beta$-D-glucopyranoside $(\mathbf{1 9}, 75 \mathrm{mg})$ in THF $(4 \mathrm{~mL})$. The reaction mixture was stirred at reflux temperature for $5.5 \mathrm{~h}$. The mixture was concentrated in vacuo to afford crude 2-propynyl

(2,3,4-tri-O-acetyl-6-[acetyl(tert-butoxycarbonyl)amino]-6-deoxy- $\beta$-D-glucopyranosyl)-(1 $\rightarrow 4)$-2,3di- $O$-acetyl-6-[acetyl(tert-butoxycarbonyl)amino]-6-deoxy- $\beta$-D-glucopyranoside (20, $127.5 \mathrm{mg}$; 
344

345

346

347

348

349

350

351

352

353

354

355

356

357

358

359

360

361

362

363

364

365

366

367

368

369

370

371

372

373

374

375

376

377

378

379

$\mathrm{MW}=872.9$, MALDI-TOF MS: $\left.m / z[M+\mathrm{Na}]^{+}=895.4\right)$.

Sodium methoxide (28\%) in methanol $(13 \mu \mathrm{L})$ was added to a solution of crude compound 20 (97.3 $\mathrm{mg})$ in methanol $(2 \mathrm{~mL})$ and dichloromethane $(1 \mathrm{~mL})$. The reaction mixture was stirred at room temperature for $6 \mathrm{~h}$. The mixture was neutralized with Amberlyst $\mathrm{H}^{+}$. After filtration of the Amberlyst $\mathrm{H}^{+}$and washing with methanol, the combined filtrate and washings were concentrated to dryness to produce crude product. The crude product was purified by preparative thin-layer chromatography (PTLC; eluent: methanol/dichloromethane=1/9, v/v) to afford 2-propynyl (6-(tert-butoxycarbonyl)amino-6-deoxy- $\beta$-D-glucopyranosyl)-(1 $\rightarrow 4)-6$ - $($ tert-butoxycarbonyl)amino -6-deoxy- $\beta$-D-glucopyranoside $\left(\mathbf{2 1}, 49.1 \mathrm{mg}, \mathrm{MW}=578.6\right.$; MALDI-TOF MS: $m / z[M+\mathrm{Na}]^{+}=$ 601.4).

Compound $21(49.1 \mathrm{mg})$ was then dissolved in acetic anhydride $(0.3 \mathrm{~mL})$ and pyridine $(2 \mathrm{~mL})$. The reaction mixture was stirred at $60{ }^{\circ} \mathrm{C}$ for $2 \mathrm{~h}$ and concentrated azeotropically with toluene to give 2-propynyl

(2,3,4-tri- $O$-acetyl-6-(tert-butoxycarbonyl)amino-6-deoxy- $\beta$-D-glucopyranosyl)-( $1 \rightarrow 4)$-2,3-di- $O$-ac etyl-6-(tert-butoxycarbonyl)amino-6-deoxy- $\beta$-D-glucopyranoside (10, $67.9 \mathrm{mg}, 77 \%$ yield from compound 19).

${ }^{1} \mathrm{H}-\mathrm{NMR}\left(\mathrm{CDCl}_{3}\right): \delta 1.44,1.46\left(18 \mathrm{H}, \mathrm{COOC}\left(\mathrm{CH}_{3}\right)_{3}\right), 1.98,2.04,2.04,2.06,2.08\left(15 \mathrm{H}, \mathrm{m}, \mathrm{COCH}_{3}\right)$, 2.49 (t, 1H, $J=2.5$ Hz, $\mathrm{CH}_{2} \mathrm{CCH}$ ), 3.31-3.36 (m, 3H, H6, H6', H6'), 3.50-3.54 (m, 2H, H5, H5'), 3.63-3.65 (m, 1H, H6'), 3.69 (t, 1H, J=9.5 Hz, H4), 4.31-4.39 (2H, m, $\left.\left(\mathrm{C}_{2}{ }_{2} \mathrm{CCH}\right)\right), 4.71$ (d, 1H, $J=7.0 \mathrm{~Hz}, \mathrm{H1}$ ), 4.76 (broad d, 1H, $J=8.0$ Hz, H1'), 4.90-4.96 (m, 4H, H2, H2', H4', NH), 5.17 (t, 1H, $J=9.5 \mathrm{~Hz}, \mathrm{H} 3$ '), 5.20 (t, 1H, $J=9.5 \mathrm{~Hz}, \mathrm{H} 3), 5.15-5.22(1 \mathrm{H}, \mathrm{NH})$

${ }^{13} \mathrm{C}-\mathrm{NMR}\left(\mathrm{CDCl}_{3}\right): \delta 20.6,20.7,20.7,20.8,20.8\left(\mathrm{COCH}_{3}\right), 28.3,28.4\left(\mathrm{COOC}\left(\mathrm{CH}_{3}\right)_{3}\right), 40.6,40.7$ (C6, C6'), 56.4 ( $\left.\underline{\mathrm{CH}}_{2} \mathrm{CCH}\right), 68.7$ (broad, C4'), 71.4 (C2), 71.6 (C2'), 72.1 (broad, C3), 72.9 (C3'), 73.0 (C5 or C5'), 73.9 (broad, $\mathrm{C} 5$ or C5'), 75.4 ( $\mathrm{CH}_{2} \mathrm{C} \underline{\mathrm{CH}}$ ), 75.9 (broad, C4), 78.3 ( $\left.\mathrm{CH}_{2} \underline{\mathrm{CCH}}\right), 79.8$, 79.8 (broad, $\left.\mathrm{COO} \underline{C}\left(\mathrm{CH}_{3}\right)_{3}\right), 98.5$ (C1), 99.2 (broad, C1'), $155.7\left(\mathrm{COOC}\left(\mathrm{CH}_{3}\right)_{3}\right), 169.4,169.6$, $169.7,170.2\left(\mathrm{COCH}_{3}\right)$

$\mathrm{Mw}=788.8$ MALDI-TOF MS: $m / z[\mathrm{M}+\mathrm{Na}]^{+}=811.5, \mathrm{~m} / z[\mathrm{M}+\mathrm{K}]^{+}=827.4$

2,3,4,6-Tetra- $O$-acetyl- $\beta$-D-glucopyranosyl-( $1 \rightarrow 4)$-2,3,6-tri- $O$-acetyl- $\beta$-D-glucopyranosyl azide (22):

Compound 22 was prepared according to the method in our previous report (H. Kamitakahara \& Nakatsubo, 2005).

$\beta$-D-Glucopyranosyl- $(1 \rightarrow 4)-\beta$-D-glucopyranosyl azide (23):

Compound 23 was prepared according to the method in previous reports (Schamann \& Schafer, 2003; Ying \& Gervay-Hague, 2003). 
$381 \quad \beta$-D-Glucopyranosiduronosyl-(1 $\rightarrow 4)-\beta$-D-glucopyranosiduronosyl azide (24):

382 Potassium bromide $(18.8 \mathrm{mg})$ and TEMPO $(16.7 \mathrm{mg})$ were added to a solution of

$383 \beta$-D-glucopyranosyl-( $1 \rightarrow 4)-\beta$-D-glucopyranosyl azide $(\mathbf{2 3}, 290 \mathrm{mg})$ in sat. aq. sodium

384 hydrogencarbonate $(3 \mathrm{~mL})$. Sodium hypochlorite $(\mathrm{NaOCl}, 3.9 \mathrm{~mL})$ was then added to the reaction 385 mixture. The mixture was stirred at $0{ }^{\circ} \mathrm{C}$ for $1 \mathrm{~h}$. TEMPO $(8 \mathrm{mg})$ and $\mathrm{NaOCl}(3.9 \mathrm{~mL})$ were further 386 added to the reaction mixture. After being stirred at $4{ }^{\circ} \mathrm{C}$ for one day, the reaction mixture was 387 extracted with distilled water and washed with dichloromethane four times. The aqueous layer was 388 adjusted to $\mathrm{pH} 2$ with $2 \mathrm{~N} \mathrm{HCl}$, concentrated, and diluted with distilled water. This concentration 389 and dilution cycle was repeated several times until the color of the solution turned from yellow to 390 colorless. The aqueous layer was finally concentrated to dryness. The insoluble part was filtered off 391 and washed with methanol. The combined filtrate and washings were concentrated to dryness. This 392 procedure was repeated three times to give

$393 \beta$-D-glucopyranosiduronosyl-(1 $\rightarrow 4)-\beta$-D-glucopyranosiduronosyl azide $(\mathbf{2 4}, 278.7 \mathrm{mg}$ ) (Schamann 394 \& Schafer, 2003; Ying \& Gervay-Hague, 2003). The carboxylic acid moieties of crude compound $395 \quad 24$ were esterified without further purification.

Methyl [(methyl $\beta$-D-glucopyranosyluronate)-(1 $\rightarrow 4)-\beta$-D-glucopyranosyl azide]uronate $(\mathbf{2 5})$ : 2,2-Dimethoxypropane $(1.5 \mathrm{~mL})$ and one drop of conc. $\mathrm{HCl}$ were added at room temperature to a solution of $\beta$-D-glucopyranosiduronosyl-(1 $\rightarrow 4)$ - $\beta$-D-glucopyranosiduronosyl azide $(\mathbf{2 4}, 278.7 \mathrm{mg})$ in methanol $(15 \mathrm{~mL})$. The reaction mixture was stirred for one day and concentrated to dryness to give methyl [(methyl $\beta$-D-glucopyranosyluronate)-( $1 \rightarrow 4)$ - $\beta$-D-glucopyranosyl azide]uronate $(\mathbf{2 5}$, $273.3 \mathrm{mg}$ ) (Schamann \& Schafer, 2003). Crude compound 25 was acetylated without further purification.

Methyl $[$ (methyl

2,3,4-tri- $O$-acetyl- $\beta$-D-glucopyranosyluronate)-( $1 \rightarrow 4)$-2,3-di- $O$-acetyl- $\beta$-D-glucopyranosyl azide]uronate (11): $\mathrm{mg}$ ) was dispersed in acetic anhydride $(4 \mathrm{~mL})$ with sodium acetate $(70.8 \mathrm{mg})$. The reaction mixture was stirred at $60^{\circ} \mathrm{C}$ overnight. The organic layer was extracted with ethyl acetate, washed with

411 distilled water twice, aq. sodium hydrogen carbonate three times, and brine, and concentrated to 412 dryness. The crude product was purified by silica gel column chromatography (eluents: ethyl 413 acetate $/ n$-hexane $=1 / 1, \mathrm{v} / \mathrm{v}$; methanol/dichloromethane $=1 / 49, \mathrm{v} / \mathrm{v}$ ) and by PTLC (eluent: ethyl 414 acetate $/ n$-hexane $=1 / 1, \mathrm{v} / \mathrm{v})$ to give methyl [(methyl 415 2,3,4-tri- $O$-acetyl- $\beta$-D-glucopyranosyluronate)-(1 $\rightarrow 4)$-2,3-di- $O$-acetyl- $\beta$-D-glucopyranosyl 
416

417

418

419

420

421

422

423

424

425

426

427

428

429

430

431

432

433

434

435

436

437

438

439

440

441

442

443

444

445

446

447

448

449

450

451

azide]uronate (11, $70 \mathrm{mg}$, total yield $11 \%$ from compound $\mathbf{2 3})$.

${ }^{1} \mathrm{H}-\mathrm{NMR}\left(\mathrm{CDCl}_{3}\right): \delta 1.99,2.01,2.01,2.06,2.07\left(15 \mathrm{H}, \mathrm{COCH}_{3}\right), 3.73$ (s, 3H, C6'OOC$\left.\underline{H}_{3}\right), 3.87$ (s, $\left.3 \mathrm{H}, \mathrm{C} 6 \mathrm{OOC} \underline{H}_{3}\right), 4.0$ (d, 2H, $J=10 \mathrm{~Hz}, \mathrm{H} 5$ and H5'), 4.14 (t, 1H, J=9.5 Hz, H4), 4.63 (d, 1H, $J=8.0$ Hz, H1'), 4.67 (d, 1H, J=8.5 Hz, H1), 4.88 (t, 2H, J=9.5 Hz, H2 and H2'), 5.13 (t, 1H, $J=10.0 \mathrm{~Hz}$, H4'), 5.19 (t, 1H, $J=9.5 \mathrm{~Hz}, \mathrm{H} 3$ '), 5.20 (t, 1H, $J=9.5 \mathrm{~Hz}, \mathrm{H} 3$ )

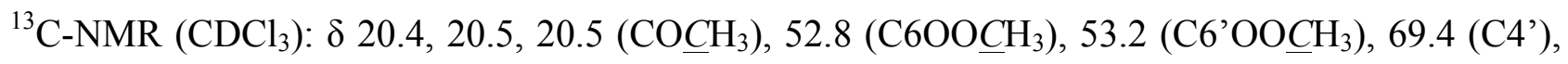
70.5 (C2'), 71.0 (C2), 71.5 (C3), 72.0 (C3'), 72.6 (C5'), 75.7 (C5), 76.4 (C4), 88.4 (C1), 100.4 $\left(\mathrm{C}^{\prime}{ }^{\prime}\right), 166.7\left(\underline{\mathrm{C}^{\prime} \mathrm{OOCH}_{3}}\right), 167.1\left(\underline{\mathrm{C}} 6 \mathrm{OOCH}_{3}\right), 169.0,169.3,169.3,170.0,170.1\left(\underline{\mathrm{COCH}}_{3}\right)$ $\mathrm{Mw}=633.5$ MALDI-TOF MS: $\mathrm{m} / z[\mathrm{M}+\mathrm{Na}]^{+}=656.4, \mathrm{~m} / z[\mathrm{M}+\mathrm{K}]^{+}=672.3$

Tri- $O$-methyl cellulosyl azide (7):

Compound 7 was prepared according to the method in our previous paper (Hiroshi Kamitakahara et al., 2016).

Propargyl tri- $O$-methyl celluloside (8):

Compound $\mathbf{8}$ was prepared according to the method in our previous paper (Hiroshi Kamitakahara et al., 2016).

1-(2,3,6-Tri- $O$-methyl-cellulosyl)-4-(2,3,4,6-tetra- $O$-acetyl- $\beta$-D-glucopyranosyl-( $1 \rightarrow 4)-2,3,6$-tri- $O$ acetyl- $\beta$-D-glucopyranosyloxymethyl)-1H-1,2,3-triazole (4):

$\mathrm{Cu}$ (I) $\mathrm{Br}(26.7 \mathrm{mg}, \mathrm{MW}=143.45,186 \mathrm{mmol}, 10$ equiv. $)$, sodium ascorbate $(73.8 \mathrm{mg} / 0.09 \mathrm{~mL}, 20$ equiv., $4 \mathrm{M}$ in $\mathrm{H}_{2} \mathrm{O}$ ), and $N, N, N^{\prime}, N^{\prime \prime}, N^{\prime \prime}$-pentamethyldiethylenetriamine (PMDETA, MW = 173.3, $d$ $=0.83 \mathrm{~g} / \mathrm{mL}, 0.04 \mathrm{~mL}, 0.0332 \mathrm{~g}, 192 \mathrm{mmol}, 10$ equiv.) were added at room temperature to a solution of 2-propynyl

(2,3,6-tri-O-acetyl- $\beta$-D-glucopyranosyl)-(1 $\rightarrow 4)$-2,3,6-tri- $O$-acetyl- $\beta$-D-glucopyranoside (9) (25.1 $\mathrm{mg}, \mathrm{MW}=674.6,37.2 \mathrm{mmol}, 2.0$ equiv.) and tri- $O$-methyl cellulosyl azide $\left(7,98.5 \mathrm{mg}, M_{\mathrm{n}}=\right.$ $5.37 \times 10^{3}, D P_{\mathrm{n}}=26.3,18.4 \mathrm{mmol}, 1.0$ equiv. $)$ in $\mathrm{MeOH} / \mathrm{CH}_{2} \mathrm{Cl}_{2}(4 \mathrm{~mL}, 1 / 4, \mathrm{v} / \mathrm{v})$. Distilled water $(0.2 \mathrm{~mL})$ was added. The reaction mixture was stirred at room temperature for four days under a nitrogen atmosphere. The mixture was concentrated and passed through a silica gel chromatography column eluted with $20 \% \mathrm{MeOH} / \mathrm{CH}_{2} \mathrm{Cl}_{2}$ to give the crude product. The crude product was purified by silica gel column chromatography (eluent: $\mathrm{EtOAc} \rightarrow 20 \% \mathrm{MeOH} / \mathrm{CH}_{2} \mathrm{Cl}_{2}$ ) to give 1-(2,3,6-tri- $O$-methyl-cellulosyl)-4-(2,3,4,6-tetra- $O$-acetyl- $\beta$-D-glucopyranosyl-( $1 \rightarrow 4)-2,3,6$-tri- $O$-a cetyl- $\beta$-D-glucopyranosyloxymethyl)-1 $H$-1,2,3-triazole $(4,86.8 \mathrm{mg}, 78.5 \%$ yield $)$.

${ }^{1} \mathrm{H}-\mathrm{NMR}\left(500 \mathrm{MHz}, \mathrm{CDCl}_{3}\right): \delta$ 1.97, 1.99, 2.02, 2.07, $2.13\left(\mathrm{COCH}_{3}\right), 2.94\left(\mathrm{t}, J=8.5 \mathrm{~Hz}, \mathrm{H} 2_{\mathrm{Me}}\right.$ (internal)), 3.20 (t, 1H, J=9.0 Hz, H3 $3_{\mathrm{Me}}$ (internal)), 3.27 (m, J=9.5 Hz, H5 $5_{\mathrm{Me}}$ (internal)), 3.37 (s, $\left.\mathrm{OCH}_{3}\right), 3.52\left(\mathrm{~s}, \mathrm{OCH}_{3}\right), 3.56\left(\mathrm{~s}, \mathrm{OCH}_{3}\right), 3.6-3.9\left(\mathrm{H}_{\mathrm{Ac}}, \mathrm{H}_{4 \mathrm{Ac}}, \mathrm{H} 5{ }_{\mathrm{Ac}}\right), 3.65\left(\mathrm{H} 6_{\mathrm{Me}}\right.$ (internal)), $3.68(\mathrm{t}$, 
452

453

454

455

456

457

458

459

460

461

462

463

464

465

466

467

468

469

470

471

472

473

474

475

476

477

478

479

480

481

482

483

484

485

486

487

$J=9.0, \mathrm{H}_{\mathrm{Me}}$ (internal)), $3.75\left(\mathrm{~m}, \mathrm{H} 6_{\mathrm{Me}}\right.$ (internal)), $3.99\left(\mathrm{~m}, J=10.0 \mathrm{~Hz}, \mathrm{H} 5 \alpha_{\mathrm{Me}}, \alpha\right.$-anomer), 4.04 (dd, $1 \mathrm{H}, J=2.0 \mathrm{~Hz}, J=12.5 \mathrm{~Hz}, \mathrm{H6}^{\prime}{ }_{\mathrm{Ac}}$ ), 4.11 (dd, $1 \mathrm{H}, J=5.0 \mathrm{~Hz}, J=12.0 \mathrm{~Hz}, \mathrm{H}_{\mathrm{Ac}}$ ), 4.17 (t, $J=7.5 \mathrm{~Hz}$, $\mathrm{H} 3 \alpha_{\mathrm{Me}}, \alpha$-anomer), 4.33 (d, $J=8.0 \mathrm{~Hz}, \mathrm{H} 1_{\mathrm{Me}}$ (internal)), $4.37\left(\mathrm{H}^{\prime}{ }_{\mathrm{Ac}}\right), 4.50$ (d, J=8.0 Hz, H1 ' ${ }_{\mathrm{Ac}}$ ), 4.54 (dd, $J=1.5 \mathrm{~Hz}, J=11.5, \mathrm{H} 6_{\mathrm{Ac}}, \beta$-anomer), 4.55 (dd, $J=2.0 \mathrm{~Hz}, J=11.5 \mathrm{~Hz}, \mathrm{H} 6_{\mathrm{Ac}}, \alpha$-anomer), 4.60 (d, $J=8.0 \mathrm{~Hz}, \mathrm{H} 1_{\mathrm{Ac}}, \beta$-anomer of methylcellulose), 4.61 (d, $J=8.0 \mathrm{~Hz}, \mathrm{H} 1_{\mathrm{Ac}}, \beta$-anomer of methylcellulose), 4.81 (d, $1 \mathrm{H}, J=12.5 \mathrm{~Hz}, \mathrm{OC} \underline{H}_{2}$-triazole), 4.87-4.94 (d, OC$\underline{H}_{2}$-triazole), 4.87-4.94 $\left(\mathrm{H} 2_{\mathrm{Ac}}, \mathrm{H} 2{ }^{\prime}{ }_{\mathrm{Ac}}\right), 5.05$ (t, $\left.J=9.5, \mathrm{H} 4{ }^{\prime}{ }_{\mathrm{Ac}}\right), 5.13$ (t, $\left.J=9.5 \mathrm{~Hz}, \mathrm{H} 3_{\mathrm{Ac}}, \mathrm{H} 3{ }^{\prime}{ }_{\mathrm{Ac}}\right), 5.44$ (d, 1H, J=9.0 Hz, $\left.\mathrm{H} 1 \beta_{\mathrm{Me}}\right), 6.15$ (d, $\left.J=5.5 \mathrm{~Hz}, \mathrm{H} 1 \alpha_{\mathrm{Me}}\right), 7.69$ (s, triazole, $\beta$-anomer), 7.70 (s, triazole, $\alpha$-anomer) $(\alpha / \beta$ ratio $=2 / 1)$

${ }^{13} \mathrm{C}-\mathrm{NMR}\left(125 \mathrm{MHz}, \mathrm{CDCl}_{3}\right): \delta$ 20.4, 20.5, 20.6, $20.8\left(\mathrm{COCH}_{3}\right), 59.1\left(\mathrm{OCH}_{3}\right), 60.2\left(\mathrm{OCH}_{3}\right), 60.5$ $\left(\mathrm{O}_{\underline{C}} \mathrm{H}_{3}\right), 61.6,61.7\left(\mathrm{C}^{\prime}{ }_{\mathrm{Ac}}\right), 61.8\left(\mathrm{C}_{\mathrm{Ac}}\right), 62.8\left(\mathrm{OC} \underline{H}_{2}\right.$-triazole $), 67.7\left(\mathrm{C}^{\prime}{ }_{\mathrm{Ac}}\right), 70.2\left(\mathrm{C}_{\mathrm{Me}}\right.$ (internal)), $71.3\left(\mathrm{C} 2_{\mathrm{Ac}}\right.$ or $\left.\mathrm{C} 2{ }^{\prime}{ }_{\mathrm{Ac}}\right), 71.5\left(\mathrm{C} 2_{\mathrm{Ac}}\right.$ or $\left.\mathrm{C} 2{ }^{\prime}{ }_{\mathrm{Ac}}\right), 71.9,72.0\left(\mathrm{C}_{\mathrm{Ac}}\right), 72.3\left(\mathrm{C} 5{ }^{\prime}{ }_{\mathrm{Ac}}\right), 72.7\left(\mathrm{C} 3_{\mathrm{Ac}}\right.$ or $\left.\mathrm{C} 3{ }^{\prime}{ }_{\mathrm{Ac}}\right)$, $72.8\left(\mathrm{C}_{\mathrm{Ac}}\right.$ or $\left.\mathrm{C} 3{ }^{\prime}{ }_{\mathrm{Ac}}\right), 73.0,73.3,73.7,74.8\left(\mathrm{C}_{\mathrm{Me}}\right.$ (internal)), $76.2\left(\mathrm{C}_{\mathrm{Ac}}\right.$ (reducing end)), 77.4 $\left(\mathrm{C} 4_{\mathrm{Me}}\right.$ (internal)), $77.8\left(\mathrm{C}_{\mathrm{Me}}\right.$ (reducing end)), 79.3, 79.5, $81.0\left(\mathrm{C} 3 \alpha_{\mathrm{Me}}\right), 82.1\left(\mathrm{C} 2_{\mathrm{Me}}\right.$ (reducing end)), $83.3\left(\mathrm{C} 1 \alpha_{\mathrm{Me}}\right), 83.4\left(\mathrm{C} 2_{\mathrm{Me}}\right.$ (internal)), 83.7, 83.7, 84.0, 84.8, $85.0\left(\mathrm{C} 3_{\mathrm{Me}}\right.$ (internal)), $85.3\left(\mathrm{C} 4_{\mathrm{Me}}\right.$ (reducing end)), 86.1, 86.9, $87.3\left(\mathrm{C} 1 \beta_{\mathrm{Me}}(\right.$ reducing end $\left.)\right), 99.6\left(\mathrm{C}_{\mathrm{Ac}}\right), 100.7\left(\mathrm{C} 1^{\prime}{ }_{\mathrm{Ac}}\right), 103.1\left(\mathrm{Cl}_{\mathrm{Me}}\right.$ (internal)), $103.7\left(\mathrm{Cl}_{\mathrm{Me}}\right), 124.5$ (triazole $\left.C \mathrm{H}\right), 143.2\left(\mathrm{O}-\mathrm{CH}_{2}-\mathrm{C}=\right), 168.9,169.2,169.6,169.7,170.2$, $170.3,170.4\left(\mathrm{COCH}_{3}\right)$

1-(2,3,6-Tri- $O$-methyl-cellulosyl)-4-[ $\beta$-D-glucopyranosyl-( $1 \rightarrow 4)$ - $\beta$-D-glucopyranosyloxymethyl]-1 $H$-1,2,3-triazole (1):

Sodium methoxide (28\%) in methanol $(0.02 \mathrm{~mL}, 10$ equiv. per AGU) was added at room temperature to a solution of

1-(2,3,6-tri- $O$-methyl-cellulosyl)-4-(2,3,4,6-tetra- $O$-acetyl- $\beta$-D-glucopyranosyl-( $1 \rightarrow 4)-2,3,6$-tri- $O$-a cetyl- $\beta$-D-glucopyranosyloxymethyl)-1H-1,2,3-triazole $(4,76.3 \mathrm{mg})$ in $\mathrm{MeOH}(3 \mathrm{~mL})$ and THF (3.5 $\mathrm{mL}$ ). The mixture was stirred overnight at room temperature. The solution was neutralized with Amberlyst $\mathrm{H}^{+}$. The Amberlyst $\mathrm{H}^{+}$was filtered off and washed with $\mathrm{MeOH}$. The combined filtrate and washings were concentrated to dryness to give

1-(2,3,6-tri- $O$-methyl-cellulosyl)-4-[ $\beta$-D-glucopyranosyl-( $1 \rightarrow 4)$ - $\beta$-D-glucopyranosyloxymethyl]- $1 H$ -1,2,3-triazole (1, $79.3 \mathrm{mg}$, quantitative yield).

${ }^{1} \mathrm{H}-\mathrm{NMR}\left(500 \mathrm{MHz}, \mathrm{D}_{2} \mathrm{O}\right): \delta 3.03\left(\mathrm{t}, J=8.5 \mathrm{~Hz}, \mathrm{H} 2_{\mathrm{Me}}\right.$ (internal)), $3.29\left(\mathrm{~s}, \mathrm{OCH}_{3}\right), 3.34(\mathrm{t}, 1 \mathrm{H}, J=8.0$ $\mathrm{Hz}, \mathrm{H} 3_{\mathrm{Me}}$ (internal)), $3.46\left(\mathrm{~s}, \mathrm{OCH}_{3}\right), 3.47\left(\mathrm{~s}, \mathrm{OCH}_{3}\right), 4.32$ (d, J=7.0 Hz, H1 Me (internal)), 4.38 (d, $J=8.0 \mathrm{~Hz}), 4.46$ (d, $J=7.5 \mathrm{~Hz}), 4.46(\mathrm{~d}, J=8.0 \mathrm{~Hz}), 4.67,4.80$ (d, $J=13.0 \mathrm{~Hz}, \mathrm{OC} \underline{H}_{2}$-triazole), 4.90 (d, $J=12.5 \mathrm{~Hz}, \mathrm{OC} \underline{H}_{2}$-triazole), 5.68 (d, $\left.1 \mathrm{H}, J=9.5 \mathrm{~Hz}, \mathrm{H} 1 \beta_{\mathrm{Me}}\right), 6.42$ (d, $\left.J=5.5 \mathrm{~Hz}, \mathrm{H} 1 \alpha_{\mathrm{Me}}\right), 8.16,8.24$, $8.32(\mathrm{~s}$, triazole $\mathrm{CH})$

${ }^{13} \mathrm{C}-\mathrm{NMR}\left(125 \mathrm{MHz}, \mathrm{D}_{2} \mathrm{O}\right): \delta 58.3\left(\mathrm{OCH}_{3}\right.$ (internal)), 58.5, 58.8, $58.9\left(\mathrm{OC}_{\mathrm{CH}}\right.$ (internal)), 59.8, 
488

489

490

491

492

493

494

495

496

497

498

499

500

501

502

503

504

505

506

507

508

509

510

511

512

513

514

515

516

517

518

519

520

521

522

523

$60.3\left(\mathrm{OCH}_{3}\right.$ (internal)), 60.5, 61.1, 64.0, 68.8, 69.4, 69.8 ( $\mathrm{C6}_{\mathrm{Me}}$ (internal)), 70.4, 70.7, 72.7, 73.1, $73.6\left(\mathrm{C} 5_{\mathrm{Me}}\right.$ (internal)), 74.2, 74.8, 75.4, 75.7, 76.0 (C4 $4_{\mathrm{Me}}$ (internal)), 76.9, 78.4, 82.1 (C2 $\mathrm{Me}_{\mathrm{e}}$ (internal)), 82.5, $83.0\left(\mathrm{C}_{\mathrm{Me}}\right.$ (internal)), 85.0, $101.3\left(\mathrm{C}_{\mathrm{OH}}\right), 102.4\left(\mathrm{C}_{\mathrm{Me}}\right.$ (internal)), $102.5\left(\mathrm{C} 1^{\prime}{ }_{\mathrm{OH}}\right)$ GPC analysis of acetylated compound 1: $M_{\mathrm{n}}=4.9 \times 10^{3}, M_{\mathrm{w}} / M_{\mathrm{n}}=1.7, D P_{\mathrm{n}}=23$ (including DP of hydrophilic segment)

1-(Tri-O-methyl-cellulosyl)-4-(2,3,4-tri-O-acetyl-6-(tert-butoxycarbonyl)amino-6-deoxy- $\beta$-D-gluco pyranosyl)-(1 $\rightarrow 4)$-2,3-di-O-acetyl-6-(tert-butoxycarbonyl)amino-6-deoxy- $\beta$-D-glucopyranosyloxy methyl)-1H-1,2,3-triazole (5):

$\mathrm{CuSO}_{4} \cdot \mathrm{H}_{2} \mathrm{O}(34.0 \mathrm{mg}, \mathrm{MW}=249.69,136 \mathrm{mmol}, 10$ equiv. $)$, sodium ascorbate $(54.0 \mathrm{mg} / 68 \mu \mathrm{L}, 20$ equiv., $4 \mathrm{M}$ in $\mathrm{H}_{2} \mathrm{O}$ ), and PMDETA ( $\mathrm{MW}=173.3, d=0.83 \mathrm{~g} / \mathrm{mL}, 28 \mu \mathrm{L}, 23.2 \mathrm{mg}, 134 \mathrm{mmol}, 10$ equiv.) were added to a solution of 2-propynyl

[2,3,4-tri-O-acetyl-6-(tert-butoxycarbonyl)amino-6-deoxy- $\beta$-D-glucopyranosyl]-( $1 \rightarrow 4)$-2,3-di- $O$-ac etyl-6-(tert-butoxycarbonyl)amino-6-deoxy- $\beta$-D-glucopyranoside (10, $32.2 \mathrm{mg}, \mathrm{MW}=788.8,40.8$ mmol, 3.0 equiv.) and tri- $O$-methyl cellulosyl azide (7, $100 \mathrm{mg}, M_{\mathrm{n}}=7.34 \times 10^{3}, D P_{\mathrm{n}}=35.8,13.6$ mmol, 1.0 equiv. $)$ in tetrahydrofuran $(3 \mathrm{~mL})$. The reaction mixture was stirred at $50{ }^{\circ} \mathrm{C}$ overnight in a nitrogen atmosphere. The mixture was concentrated and passed through a silica gel chromatography column eluted with $10 \% \mathrm{MeOH} / \mathrm{CH}_{2} \mathrm{Cl}_{2}$ to give the crude product. The crude product was purified by silica gel column chromatography (eluent: EtOAc $\rightarrow 10 \% \mathrm{MeOH} / \mathrm{CH}_{2} \mathrm{Cl}_{2}$ ) to give

1-(tri- $O$-methyl-cellulosyl)-4-(2,3,4-tri-O-acetyl-6-(tert-butoxycarbonyl)amino-6-deoxy- $\beta$-D-glucop yranosyl)-(1 $\rightarrow 4)$-2,3-di-O-acetyl-6-(tert-butoxycarbonyl)amino-6-deoxy- $\beta$-D-glucopyranosyloxym ethyl)-1H-1,2,3-triazole (5, $103.2 \mathrm{mg}, 93 \%$ yield).

${ }^{1} \mathrm{H}-\mathrm{NMR}\left(500 \mathrm{MHz}, \mathrm{CDCl}_{3}\right): \delta 1.44,1.47\left(\mathrm{CH}_{3}\right.$ (NHBoc)), 1.97, 1.98, 2.00, 2.02, 2.02, 2.04, 2.05 , $2.07\left(\mathrm{CH}_{3}(\mathrm{OAc})\right), 2.95$ (t, $J=8.0 \mathrm{~Hz}, \mathrm{H} 2_{\mathrm{Me}}$ (internal)), 3.11 (t, $\left.J=9.0 \mathrm{~Hz}, \mathrm{H} 3_{\mathrm{Me}}\right), 3.21(\mathrm{t}, 1 \mathrm{H}, J=9.0$ $\mathrm{Hz}, \mathrm{H} 3_{\mathrm{Me}}$ (internal)), 3.29 (m, J=9.0 Hz, H5 ${ }_{\mathrm{Me}}$ (internal)), 3.3-3.4 (H6, H6', H6'), 3.6-3.65 (H6'), $3.38\left(\mathrm{OCH}_{3}\right), 3.54\left(\mathrm{OCH}_{3}\right), 3.58\left(\mathrm{OCH}_{3}\right), 3.62-3.72\left(\mathrm{H} 6 \mathrm{Me}\right.$ (internal)), $3.69\left(\mathrm{t}, J=9.5 \mathrm{~Hz}, \mathrm{H} 4_{\mathrm{Me}}\right.$ (internal)), 3.73-3.81 (m, H6 $6_{\mathrm{Me}}$ (internal)), 3.40 ( $\mathrm{H} 2_{\mathrm{Me}}$, reducing-end, $\alpha$-anomer), $3.77\left(\mathrm{H} 2_{\mathrm{Me}}\right.$, reducing-end, $\alpha$-anomer), $3.77\left(\mathrm{H} 2_{\mathrm{Me}}\right.$, reducing-end, $\alpha$-anomer), $3.83\left(\mathrm{H} 2_{\mathrm{Me}}\right.$, reducing-end, $\alpha$-anomer), 3.89 (H6 $6_{\mathrm{Me}}$, reducing-end, $\alpha$-anomer), 4.04 (m, $J=9.5 \mathrm{~Hz}, \mathrm{H} 5_{\mathrm{Me}}$, reducing-end, $\alpha$-anomer), 4.15 (t, $J=7.5 \mathrm{~Hz}, \mathrm{H} 3_{\mathrm{Me}}$, reducing-end, $\alpha$-anomer), 4.34 (d, $J=7.5 \mathrm{~Hz}, \mathrm{H} 1_{\mathrm{Me}}$ (internal)), 4.39 (d, J=8.0 Hz, H1 ${ }_{\mathrm{Me}}$ ), 4.6-4.64 (d, $\left.\mathrm{H} 1_{\mathrm{Ac}}\right), 4.76$ (broad d, $1 \mathrm{H}, J=8.0 \mathrm{~Hz}, \mathrm{H} 1^{\prime}{ }_{\mathrm{Ac}}$ ), 4.75-4.94 ( $\mathrm{CH}_{2}$, triazole-alkene), 4.90-4.96 (H2 $\left.{ }_{\mathrm{Ac}}, \mathrm{H} 2{ }^{\prime}{ }_{\mathrm{Ac}}, \mathrm{H} 4{ }^{\prime}{ }_{\mathrm{Ac}}, \mathrm{NH}\right), 5.1-5.2\left(\mathrm{H}_{\mathrm{Ac}}, \mathrm{H} 3{ }^{\prime}{ }_{\mathrm{Ac}}\right), 5.46$ $\left(\mathrm{d}, J=9.0 \mathrm{~Hz}, \mathrm{H} 1 \beta_{\mathrm{Me}}\right), 6.15\left(\mathrm{~d}, J=5.0 \mathrm{~Hz}, \mathrm{H} 1 \alpha_{\mathrm{Me}}\right), 7.71,7.76(\mathrm{H}$, triazole $)$

${ }^{13} \mathrm{C}-\mathrm{NMR}\left(125 \mathrm{MHz}, \mathrm{CDCl}_{3}\right): \delta 20.6,20.63,20.65,20.7,20.8\left(\mathrm{COCH}_{3}\right), 28.4\left(\mathrm{COOC}\left(\mathrm{CH}_{3}\right)_{3}\right), 40.7$ (C6, C6'), 59.0, $59.2\left(\mathrm{OCH}_{3}\right), 59.6,60.1,60.3\left(\mathrm{OCH}_{3}\right), 60.4,60.5,60.5\left(\mathrm{OCH}_{3}\right), 60.8,62.8$ 
524 (OCH ${ }_{2}$-triazole), 68.5 (broad, $\left.\mathrm{C} 4{ }^{\prime}{ }_{\mathrm{Ac}}\right), 70.3$ (C6 ${ }_{\mathrm{Me}}$ (internal)), $71.6\left(\mathrm{C}_{\mathrm{Ac}_{\mathrm{Ac}}}\right.$ and $\left.\mathrm{C} 2^{\prime}{ }_{\mathrm{Ac}}\right), 72.1\left(\mathrm{C} 3_{\mathrm{Ac}}\right)$,

525

526

527

528

529

530

531

532

533

534

535

536

537

538

539

540

541

542

543

544

545

546

547

548

549

550

551

552

553

554

555

556

557

558

559

$72.9\left(\mathrm{C}^{\prime}{ }_{\mathrm{Ac}}\right), 73.0\left(\mathrm{C}_{\mathrm{Ac}}\right.$ or $\left.\mathrm{C} 5{ }^{\prime}{ }_{\mathrm{Ac}}\right), 73.1,73.2,73.9\left(\mathrm{broad}, \mathrm{C}_{\mathrm{Ac}}\right.$ or $\left.\mathrm{C}^{\prime}{ }_{\mathrm{Ac}}\right), 74.9\left(\mathrm{C}_{\mathrm{Me}}\right.$

(internal)), $75.5\left(\mathrm{C}_{\mathrm{Ac}}\right), 77.5\left(\mathrm{C}_{\mathrm{Me}}\right.$ (internal)), 79.8 $\left.\mathrm{COOC}\left(\mathrm{CH}_{3}\right)_{3}\right), 83.3,83.5\left(\mathrm{C} 2_{\mathrm{Me}}\right.$ (internal)), $83.7\left(\mathrm{C} 1 \alpha_{\mathrm{Me}}\right), 84.9,85.0\left(\mathrm{C} 3_{\mathrm{Me}}\right.$ (internal)), 86.1, 99.1( $\left.\mathrm{C}_{\mathrm{Ac}}\right), 99.8\left(\mathrm{C} 1^{\prime}{ }_{\mathrm{Ac}}\right), 103.2\left(\mathrm{C}_{\mathrm{Me}}\right.$ (internal)), 122.2, 124.4 (triazole $\mathrm{CH}), 143.2,144.2\left(\mathrm{O}-\mathrm{CH}_{2}-\mathrm{C}=\right), 155.8\left(\mathrm{COOC}\left(\mathrm{CH}_{3}\right)_{3}\right), 169.3,169.6,169.7$, $170.2\left(\mathrm{COCH}_{3}\right)$

1-(Tri-O-methyl-cellulosyl)-4-[(6-amino-6-deoxy- $\beta$-D-glucopyranosyl)-(1 $\rightarrow 4)-6$-amino-6-deoxy- $\beta$ D-glucopyranosyloxymethyl]-1H-1,2,3-triazole (2):

Sodium methoxide (28\%) in methanol $(5.5 \mu \mathrm{L}, 10$ equiv. per $\mathrm{AGU})$ were added at room temperature to a solution of

1-(tri- $O$-methyl-cellulosyl)-4-[2,3,4-tri- $O$-acetyl-6-(tert-butoxycarbonyl)amino-6-deoxy- $\beta$-D-glucop yranosyl-( $1 \rightarrow 4$ )-2,3-di-O-acetyl-6-(tert-butoxycarbonyl)amino-6-deoxy- $\beta$-D-glucopyranosyloxymet hyl]-1 $\mathrm{H}-1,2,3$-triazole $(\mathbf{5}, 76.3 \mathrm{mg})$ in $\mathrm{MeOH}(2 \mathrm{~mL})$, THF $(2 \mathrm{~mL})$, and $\mathrm{CH}_{2} \mathrm{Cl}_{2}(1 \mathrm{~mL})$. The mixture was stirred overnight at room temperature. The solution was neutralized with Amberlyst $\mathrm{H}^{+}$. The Amberlyst $\mathrm{H}^{+}$was filtered off and washed with $\mathrm{MeOH}$. The combined filtrate and washings were concentrated to dryness to give 1-(tri- $O$-methyl-cellulosyl)-4-[6-(tert-butoxycarbonyl)amino-6-deoxy- $\beta$-D-glucopyranosyl-(1 $\rightarrow 4)-6$ -(tert-butoxycarbonyl)amino-6-deoxy- $\beta$-D-glucopyranosyloxymethyl]-1 $H$-1,2,3-triazole (78.9 mg).

Trifluoroacetic acid $(0.5 \mathrm{~mL})$ was added at $-20{ }^{\circ} \mathrm{C}$ to a solution of

1-(tri-O-methyl-cellulosyl)-4-[6-(tert-butoxycarbonyl)amino-6-deoxy- $\beta$-D-glucopyranosyl)-(1 $\rightarrow 4)$ 6-(tert-butoxycarbonyl)amino-6-deoxy- $\beta$-D-glucopyranosyloxymethyl]-1 H-1,2,3-triazole (84 mg) in $\mathrm{CH}_{2} \mathrm{Cl}_{2}(1 \mathrm{~mL})$. The reaction mixture was stirred for $1.2 \mathrm{~h}$ at $-20{ }^{\circ} \mathrm{C}$. The mixture was concentrated to dryness at $4{ }^{\circ} \mathrm{C}$ to give the crude product. The crude product was purified by gel filtration chromatography (Sephadex LH-20) to give 1-(tri- $O$-methyl-cellulosyl)-4-[6-amino-6-deoxy- $\beta$-D-glucopyranosyl-( $1 \rightarrow 4)-6$-amino-6-deoxy- $\beta$-Dglucopyranosyloxymethyl]-1H-1,2,3-triazole $(2,82.7 \mathrm{mg}$, quantitative yield).

${ }^{1} \mathrm{H}-\mathrm{NMR}\left(500 \mathrm{MHz}, \mathrm{D}_{2} \mathrm{O}\right): \delta 3.03\left(\mathrm{t}, J=8.5 \mathrm{~Hz}, \mathrm{H} 2_{\mathrm{Me}}\right.$ (internal)), $3.34\left(\mathrm{t}, 1 \mathrm{H}, J=8.5 \mathrm{~Hz}, \mathrm{H} 3_{\mathrm{Me}}\right.$ (internal)), 3.42-3.49 $\left(\mathrm{H} 5 \mathrm{Me}\right.$ (internal)), $3.29\left(\mathrm{OCH}_{3}\right), 3.46\left(\mathrm{OCH}_{3}\right), 3.47\left(\mathrm{OC}_{3}\right), 3.57-3.68(\mathrm{H} 4 \mathrm{Me}$ (internal), $\mathrm{H} 6_{\mathrm{Me}}$ (internal)), 4.32 (d, $J=7.0 \mathrm{~Hz}, \mathrm{H} 1_{\mathrm{Me}}$ (internal)), 4.40 (d, J=8.0 Hz), 4.44 (d, $J=6.0$ $\mathrm{Hz}), 4.79-4.95\left(\mathrm{CH}_{2}\right.$, triazole-alkene), $5.69\left(\mathrm{H} 1 \beta_{\mathrm{Me}}\right.$ (reducing end), $\left.J=9 \mathrm{~Hz}\right), 6.42\left(\mathrm{H} 1 \alpha_{\mathrm{Me}}\right.$ (reducing end), $J=5.5 \mathrm{~Hz}$ ), 8.17, 8.25 (s, triazole $\mathrm{CH}$ )

${ }^{13} \mathrm{C}-\mathrm{NMR}\left(\mathrm{D}_{2} \mathrm{O}\right): \delta 40.2\left(\underline{\mathrm{C}} 6-\mathrm{NH}_{2}\right.$ or $\left.\underline{\mathrm{C}} 6-\mathrm{NH}_{2}\right), 40.5\left(\underline{\mathrm{C}} 6-\mathrm{NH}_{2}\right.$ or $\left.\underline{\mathrm{C}} 6{ }^{\prime}-\mathrm{NH}_{2}\right), 58.3\left(\mathrm{OCH}_{3}\right), 58.5,58.9$ $\left(\mathrm{OCH}_{3}\right), 59.8,60.3\left(\mathrm{OCH}_{3}\right), 69.8\left(\mathrm{C}_{\mathrm{Me}}\right.$ (internal)), 70.8, 73.0, $63.6\left(\mathrm{C}_{\mathrm{Me}}\right.$ (internal)), 74.2, 74.5, 74.7, 75.1, 75.7, 76.0 (C4 $\mathrm{Me}_{\mathrm{Me}}$ (internal)), 82.0 (C2 ${ }_{\mathrm{Me}}$ (internal)), 82.5, 83.0 (C3 $3_{\mathrm{Me}}$ (internal)) 85.0, 101.2, 101.4, $102.4\left(\mathrm{Cl}_{\mathrm{Me}}\right.$ (internal)), 102.8 
560

561

562

563

564

565

566

567

568

569

570

571

572

573

574

575

576

577

578

579

580

581

582

583

584

585

586

587

588

589

590

591

592

593

594

595

GPC analysis of acetylated compound 2: $M_{\mathrm{n}}=7.1 \times 10^{3}, M_{\mathrm{w}} / M_{\mathrm{n}}=1.6, D P_{\mathrm{n}}=33$ (including DP of hydrophilic segment)

4-(Tri-O-methyl-cellulosyloxymethyl)-1-[methyl \{(methyl

$(2,3,4$-tri- $O$-acetyl- $\beta$-D-glucopyranosyl)uronate)-( $1 \rightarrow 4)$-2,3-di- $O$-acetyl- $\beta$-D-glucopyranosyl $\}$ uronat e]-1H-1,2,3-triazole (6):

$\mathrm{Cu}$ (I) $\mathrm{Br}$ (5.6 mg, MW = 143.45, 39 mmol, 10 equiv.), sodium ascorbate ( $15.5 \mathrm{mg} / 19 \mu \mathrm{L}, 20$ equiv., $4 \mathrm{M}$ in $\mathrm{H}_{2} \mathrm{O}$ ), and PMDETA ( $\mathrm{MW}=173.3, d=0.83 \mathrm{~g} / \mathrm{mL}, 8.2 \mu \mathrm{L}, 6.8 \mathrm{mg}, 39 \mathrm{mmol}, 10$ equiv.) were added at room temperature to a solution of methyl [(methyl 2,3,4-tri- $O$-acetyl- $\beta$-D-glucopyranosyluronate)-( $1 \rightarrow 4)$-2,3-di- $O$-acetyl- $\beta$-D-glucopyranosyl azide]uronate $(11,7.4 \mathrm{mg}, \mathrm{MW}=633.5,11.7 \mathrm{mmol}, 3.0$ equiv. $)$ and propargyl tri- $O$-methyl celluloside (8, $30 \mathrm{mg}, M_{\mathrm{n}}=7.72 \times 10^{3}, D P_{\mathrm{n}}=37.5,3.9 \mathrm{mmol}, 1.0$ equiv. $)$ in $\mathrm{MeOH} / \mathrm{CH}_{2} \mathrm{Cl}_{2}(2 \mathrm{~mL}$, $1 / 4, v / v)$. The reaction mixture was stirred overnight at room temperature under a nitrogen atmosphere. The mixture was concentrated and passed through a silica gel chromatography column eluted with $10 \% \mathrm{MeOH} / \mathrm{CH}_{2} \mathrm{Cl}_{2}$ to give the crude product. The crude product was purified by silica gel column chromatography (eluent: EtOAc $\rightarrow 10 \% \mathrm{MeOH} / \mathrm{CH}_{2} \mathrm{Cl}_{2}$ ) to give 4-(tri- $O$-methyl-cellulosyloxymethyl)-1-[methyl \{(methyl $(2,3,4$-tri- $O$-acetyl- $\beta$-Dglucopyranosyl)uronate)-( $1 \rightarrow 4)$-2,3-di- $O$-acetyl- $\beta$-D-glucopyranosyl $\}$ uronate]-1 $H$-1,2,3-triazole $(\mathbf{6}$, $29.9 \mathrm{mg}, 92 \%$ yield).

${ }^{1} \mathrm{H}-\mathrm{NMR}\left(500 \mathrm{MHz}, \mathrm{CDCl}_{3}\right): \delta$ 1.86, 1.86, 2.00, 2.02, 2.02, 2.09, $2.10\left(\mathrm{COC}_{3}\right), 2.96(\mathrm{t}, J=8.5 \mathrm{~Hz}$, $\mathrm{H} 2_{\mathrm{Me}}$ (internal)), $3.22\left(\mathrm{t}, 1 \mathrm{H}, J=9.0 \mathrm{~Hz}, \mathrm{H} 3_{\mathrm{Me}}\right.$ (internal)), $3.29\left(\mathrm{~m}, J=9.0 \mathrm{~Hz}, \mathrm{H} 5_{\mathrm{Me}}\right.$ (internal)), $3.39\left(\mathrm{OCH}_{3}\right), 3.54\left(\mathrm{OCH}_{3}\right), 3.58\left(\mathrm{OCH}_{3}\right), 3.66\left(\mathrm{H}_{\mathrm{Me}}\right.$ (internal)), $3.70\left(\mathrm{t}, J=9.0 \mathrm{~Hz}, \mathrm{H} 4_{\mathrm{Me}}\right.$ (internal)), 3.77 (broad dd, $J=3,5 \mathrm{~Hz}, J=11.0 \mathrm{~Hz}, \mathrm{H} 6 \mathrm{Me}$ (internal)), 3.84 (s, 3H, C6'OOC $\underline{H}_{3}$ ), 3.85 (s, 3H, C6OOC $\left.\underline{H}_{3}\right), 4.03$ (dd, $J=3.5, J=10.0, \mathrm{H}^{\prime}{ }^{\prime}$ Ac (non-reducing end)), 4.18 (dd, $J=2.0 \mathrm{~Hz}, J=10.0$ $\mathrm{Hz}, \mathrm{H} 5_{\mathrm{Ac}}$ (reducing end)), $4.29\left(\mathrm{H} 4_{\mathrm{Ac}}\right.$ (reducing end)), 4.35 (d, $J=8.0 \mathrm{~Hz}, \mathrm{H} 1_{\mathrm{Me}}$ (internal)), 4.40 (d, $\left.J=8.0 \mathrm{~Hz}, \mathrm{H} 1_{\mathrm{Me}}\right), 4.67$ (d, $J=13.0 \mathrm{~Hz}, \mathrm{OC} \underline{H}_{2}$-triazole), 4.67-4.68 (d, $J=8, \mathrm{H} 1^{\prime}{ }_{\text {Ac }}$ (non-reducing end)), 4.82 (d, $J=13.5 \mathrm{~Hz}, \mathrm{OC} \underline{H}_{2}$-triazole), 4.85 (d, $J=13.0 \mathrm{~Hz}, \mathrm{OC} \underline{H}_{2}$-triazole), 4.90 (t, $J=8.5 \mathrm{~Hz}, \mathrm{H} 2{ }^{\prime}{ }_{\mathrm{Ac}}$ (non-reducing end)), 4.96 (d, $J=13.0 \mathrm{~Hz}, \mathrm{OCH}_{2}$-triazole), 5.03 (d, $J=3.5 \mathrm{~Hz}, \mathrm{H} 1 \alpha_{\mathrm{Me}}$ (reducing end)), $5.15\left(\mathrm{t}, J=9.5 \mathrm{~Hz}, \mathrm{H} 4{ }^{\prime}{ }_{\mathrm{Ac}}\right.$ (non-reducing end)), 5.21 ( $\mathrm{t}, J=9.5 \mathrm{~Hz}, \mathrm{H} 3{ }^{\prime}{ }_{\mathrm{Ac}}$ (non-reducing end)), 5.40 (t, $J=9.0 \mathrm{~Hz}, \mathrm{H} 2_{\mathrm{Ac}}$ (reducing end)), 5.41 ( $\mathrm{t}, J=9.0 \mathrm{~Hz}, \mathrm{H} 3_{\mathrm{Ac}}$ (reducing end)), 5.84-5.87 (d, H1 $\mathrm{Ac}$ (reducing end)), 7.81 (s, triazole $\mathrm{CH}$ )

${ }^{13} \mathrm{C}-\mathrm{NMR}\left(125 \mathrm{MHz}, \mathrm{CDCl}_{3}\right): \delta$ 20.1, 20.2, 20.4, $20.5\left(\mathrm{COCH}_{3}\right), 52.8,53.3\left(\mathrm{COOCH}_{3}\right), 58.8,59.0$, $59.1\left(\mathrm{OCH}_{3}\right), 59.3,59.6,60.1,60.1,60.3\left(\mathrm{OCH}_{3}\right), 60.4,50.4,60.5\left(\mathrm{OCH}_{3}\right), 60.6\left(\mathrm{OCH}_{2}\right.$-triazole, overlapped), 60.7, 60.8, 62.3 ( $\mathrm{O}^{\mathrm{C}} \mathrm{H}_{2}$-triazole), 69.3 (C4 ${ }^{\prime}{ }_{\mathrm{Ac}}$ (non-reducing end)), 69.9, $70.0\left(\mathrm{C} 3_{\mathrm{Ac}}\right.$ (reducing end)), 70.3 (C6 ${ }_{\mathrm{Me}}$ (internal)), 70.6, 71.0 (C2 ${ }^{\text {Ac }}$ (non-reducing end)), $71.6\left(\mathrm{C} 2_{\mathrm{Ac}}\right.$ (reducing end)), 72.0 (C3 ${ }^{\prime}$ Ac (non-reducing end)), 72.2, 72.7 (C5' ${ }^{\text {Ac }}$ (non-reducing end)), 73.2, 73.2, 74.6, 74.7, 
601

602

603

604

605

606

607

608

609

610

611

612

613

614

615

616

617

618

619

620

621

622

623

624

625

626

627

628

629

630

631

$74.9\left(\mathrm{C} 5_{\mathrm{Me}}\right.$ (internal)), $76.2\left(\mathrm{C} 4_{\mathrm{Ac}}\right.$ (reducing end $\left.)\right), 76.3\left(\mathrm{C} 5_{\mathrm{Ac}}\right.$ (reducing end $\left.)\right), 77.5\left(\mathrm{C} 4_{\mathrm{Me}}\right.$ (internal)), 83.5 (C2 $\mathrm{Me}_{\text {e }}$ (internal)), $85.0\left(\mathrm{C} 3_{\mathrm{Me}}\right.$ (internal)), $86.1\left(\mathrm{C} 1_{\mathrm{Ac}}\right.$ (reducing end)), $95.7\left(\mathrm{C} 1 \alpha_{\mathrm{Me}}\right.$ (reducing end)), $100.3\left(\mathrm{Cl}^{\prime}{ }_{\mathrm{Ac}}\right.$ (non-reducing end)), $103.2\left(\mathrm{Cl}_{\mathrm{Me}}\right.$ (internal)), $103.4\left(\mathrm{Cl}_{\mathrm{Me}}\right), 121.4$ (triazole $C \mathrm{H}), 145.1\left(\mathrm{O}-\mathrm{CH}_{2}-\mathrm{C}=\right), 166.6\left(\underline{\mathrm{C}}^{\prime}{ }^{\mathrm{Ac}} \mathrm{OOCH}_{3}\right), 166.8\left(\underline{\mathrm{C}}_{\mathrm{Ac}} \mathrm{OOCH}_{3}\right), 169.0,169.3,169.7$, $169.8,170.1\left(\mathrm{COCH}_{3}\right)$

4-(Tri- $O$-methyl-cellulosyloxymethyl)-1-[ $\beta$-D-glucopyranuronosyl-( $1 \rightarrow 4)-\beta$-D-glucopyranuronosyl] $-1 H-1,2,3$-triazole (3):

An aqueous solution of sodium hydroxide $(0.0125 \mathrm{M}, 1.5 \mathrm{~mL})$ was added at room temperature to a solution of 4-(tri- $O$-methyl-cellulosyloxymethyl)-1-[methyl (methyl 2,3,4-tri- $O$-acetyl- $\beta$-D-glucopyranuronate)-( $1 \rightarrow 4)$-2,3-di- $O$-acetyl- $\beta$-D-glucopyranuronosyl]- $1 H$ - 1,2 ,3-triazole $(6,14.4 \mathrm{mg})$ in THF $(1 \mathrm{~mL})$. The reaction mixture was stirred at room temperature for 30 min. The solution was neutralized with Amberlyst $\mathrm{H}^{+}$. The Amberlyst $\mathrm{H}^{+}$was filtered off and washed with tetrahydrofuran. The combined filtrate and washings were concentrated to dryness to give the crude product. The crude product was purified by gel filtration chromatography (Sephadex LH-20) to give 4-(tri- $O$-methylcellulosyloxymethyl)-1-[( $\beta$-D-glucopyranuronosyl)-( $1 \rightarrow 4)-\beta$-D-glucopyranuronosyl]-1H-1,2,3-triaz ole $(3,13.8 \mathrm{mg}, 99 \%$ yield $)$.

${ }^{1} \mathrm{H}-\mathrm{NMR}\left(500 \mathrm{MHz}, \mathrm{D}_{2} \mathrm{O}\right): \delta 3.03\left(\mathrm{t}, 1 \mathrm{H}, J=8.5 \mathrm{~Hz}, \mathrm{H} 2_{\mathrm{Me}}\right.$ (internal)), $3.27\left(\mathrm{H} 2_{\mathrm{Me}}\right), 3.34(\mathrm{t}, 1 \mathrm{H}$, $J=9.0 \mathrm{~Hz}, \mathrm{H} 3_{\mathrm{Me}}$ (internal)), $3.47\left(\mathrm{H} 5_{\mathrm{Me}}\right.$ (internal)), $3.29\left(\mathrm{OC}_{3}\right), 3.46\left(\mathrm{OC}_{3}\right), 3.47\left(\mathrm{OC}_{3}\right), 3.64$ $\left(\mathrm{H} 4_{\mathrm{Me}}\right.$ (internal)), 3.6-3.7 $\left(\mathrm{H} 6_{\mathrm{Me}}\right.$ (internal)), $3.77\left(\mathrm{H} 4_{\mathrm{OH}}\right), 3.96\left(\mathrm{H} 3_{\mathrm{OH}}\right), 4.02\left(\mathrm{H} 2_{\mathrm{OH}}\right), 4.31(\mathrm{~d}, J=5.5$ $\mathrm{Hz}, \mathrm{C} 1_{\mathrm{Me}}$ (internal)), 4.43 (d, $\left.J=7.0 \mathrm{~Hz}, \mathrm{H} 1_{\mathrm{Me}}\right), 4.72\left(\mathrm{OC} \underline{H}_{2}\right.$-triazole), 4.80 (d, $J=14.0 \mathrm{~Hz}$, OC $\underline{H}_{2}$-triazole), 4.88 (d, $J=13.5 \mathrm{~Hz}, \mathrm{OC}_{2}$-triazole), $5.71\left(\mathrm{~d}, J=8.5 \mathrm{~Hz}, \mathrm{H} 1_{\mathrm{OH}}\right), 8.23$ (s, triazole $\mathrm{CH}$ ) ${ }^{13} \mathrm{C}-\mathrm{NMR}\left(125 \mathrm{MHz}, \mathrm{D}_{2} \mathrm{O}\right): \delta 58.3\left(\mathrm{OCH}_{3}\right), 58.5,58.9\left(\mathrm{OCH}_{3}\right), 59.8,60.3\left(\mathrm{OCH}_{3}\right), 60.4$ ( $\mathrm{OCH}_{2}$-triazole), $62.2\left(\mathrm{OCH}_{2}\right.$-triazole $), 68.8,69.8\left(\mathrm{C}_{\mathrm{Me}}\right.$ (internal)), $71.8\left(\mathrm{C} 3_{\mathrm{OH}}\right), 73.0,73.6\left(\mathrm{C} 5_{\mathrm{Me}}\right.$ (internal)), 74.2, $74.6\left(\mathrm{C} 4_{\mathrm{OH}}\right), 75.9\left(\mathrm{C}_{\mathrm{Me}}\right.$ (internal) $), 78.0\left(\mathrm{C} 2_{\mathrm{OH}}\right), 80.3\left(\mathrm{C} 2_{\mathrm{Me}}\right), 82.1\left(\mathrm{C} 2_{\mathrm{Me}}\right.$ (internal)), 82.5, $83.0\left(\mathrm{C} 3_{\mathrm{Me}}\right.$ (internal)), $85.0\left(\mathrm{C} 3_{\mathrm{Me}}\right), 87.0\left(\mathrm{C} 1_{\mathrm{OH}}\right), 101.1,101.3,102.3\left(\mathrm{C} 1_{\mathrm{Me}}\right.$ (internal)), 124.5 (triazole $\mathrm{CH}), 144.0\left(\mathrm{O}-\mathrm{CH}_{2}-\mathrm{C}=\right.$ )

GPC analysis of acetylated compound 3: $M_{\mathrm{n}}=9.1 \times 10^{3}, M_{\mathrm{w}} / M_{\mathrm{n}}=1.9, D P_{\mathrm{n}}=43$ (including DP of hydrophilic segment)

\section{Results and Discussion}

\subsection{Synthesis of trehalose-type diblock methylcellulose analogues}

Three hydrophilic segments, nonionic, cationic, and anionic cellobiosyl residues, were coupled with hydrophobic permethylated methylcellulose segments via the Huisgen 1,3-dipolar cycloaddition to produce trehalose-type diblock methylcellulose analogues. 
632

633 3.1.1. Synthesis of hydrophilic segments

634 2-Propynyl 2,3,6-tri- $O$-acetyl- $\beta$-D-glucopyranosyl-( $1 \rightarrow 4)$-2,3,6-tri- $O$-acetyl- $\beta$-D-glucopyranoside 635 (9), 2-propynyl

636 2,3,4-tri- $O$-acetyl-6-(tert-butoxycarbonyl)amino-6-deoxy- $\beta$-D-glucopyranosyl-( $1 \rightarrow 4)$-2,3-di- $O$-acet

637 yl-6-(tert-butoxycarbonyl)amino-6-deoxy- $\beta$-D-glucopyranoside (10), and methyl [(methyl

638 2,3,4-tri- $O$-acetyl- $\beta$-D-glucopyranosyluronate)-( $1 \rightarrow 4)-2,3$-di- $O$-acetyl- $\beta$-D-glucopyranosyl

639 azide]uronate (11) were synthesized according to the synthetic routes shown in Scheme 1.

640 Compound 9 was prepared from cellobiose via cellobiose octaacetate (12) (H. Kamitakahara \&

641 Nakatsubo, 2005) and is a precursor of the nonionic hydrophilic segment that gives compound 1.

642 Huisgen 1,3-dipolar cycloaddition of compounds 7 and 9 produced compound 4. The removal of

643 the acetyl groups of compound 4 afforded compound $\mathbf{1}$. Compound $\mathbf{1}$ is analogous to

644 cellobiosyl-( $1 \rightarrow 4)$-methylcellulose, diblock methylcellulose (Nakagawa et al., 2011b), which

645 possesses thermoreversible gelation properties. Nonionic compound $\mathbf{1}$ is also a standard for cationic

646 compound 2 and anionic compound 3. In other words, nonionic compound 9 is also a standard for

647 cationic compound $\mathbf{1 0}$ and anionic compound $\mathbf{1 1 .}$ 


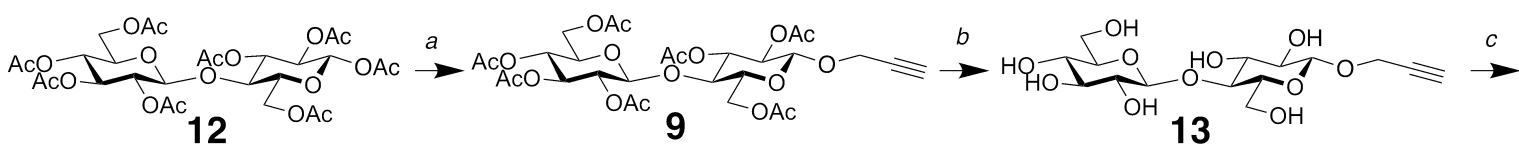

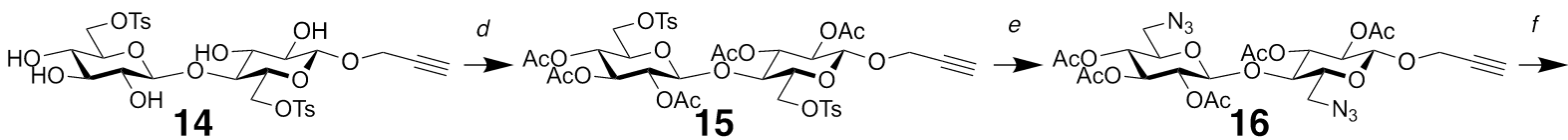

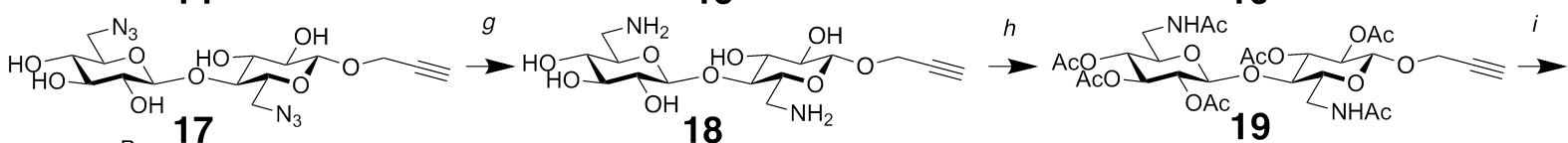

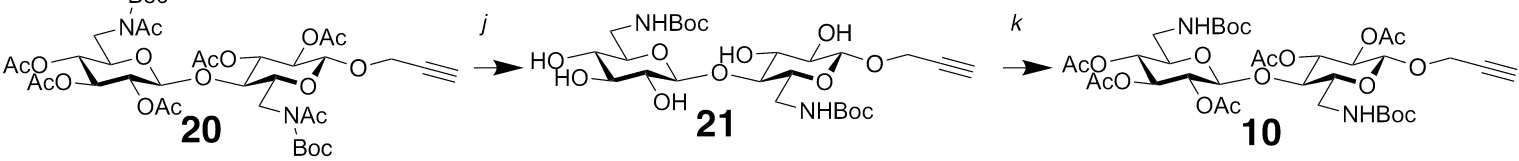

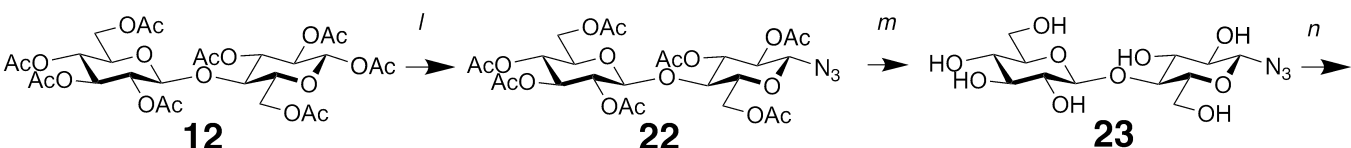

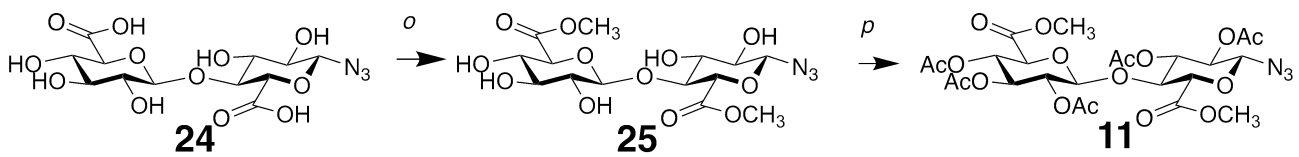

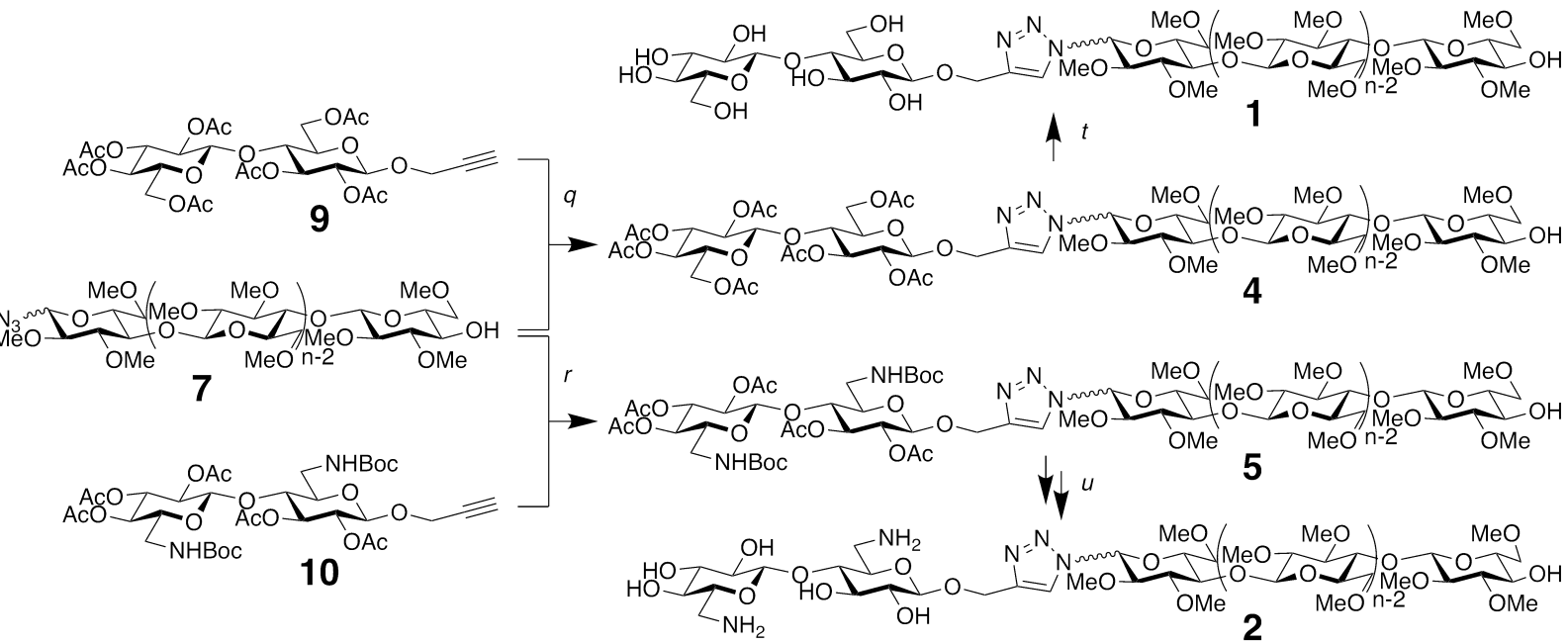
$=\underbrace{\mathrm{O}}_{\mathrm{OMe}}$

Scheme 1. Synthetic routes for trehalose-type diblock methylcellulose analogues 1, 2, and $\mathbf{3}$

a) 2-propyn-1-ol/ $\mathrm{BF}_{3} \mathrm{Et}_{2} \mathrm{O} /$ anhydrous $\mathrm{CH}_{2} \mathrm{Cl}_{2} /$ r.t./ $23 \mathrm{~h} / 99 \%$; b) $28 \% \mathrm{NaOCH}_{3}$ in $\mathrm{MeOH} / \mathrm{THF} / \mathrm{MeOH} / \mathrm{rtt} / 3 \mathrm{~h} / 96 \%$; c) $\mathrm{TsCl} /$ Pyridine/ $8^{\circ} \mathrm{C} / 2.5 \mathrm{~h} / 51 \%$; d) $\mathrm{Ac}_{2} \mathrm{O} /$ Pyridine/ r.t./overnight/ $95 \%$; e) $\mathrm{NaN}_{3} / \mathrm{DMF} / 50^{\circ} \mathrm{C} /$ overnight $/ 97 \%$; f) $28 \% \mathrm{NaOCH}_{3}$ in $\mathrm{MeOH} / \mathrm{THF} / \mathrm{MeOH} /$ r.t./overnight/ 87\%; g) $\mathrm{Ph}_{3} \mathrm{P} / \mathrm{H}_{2} \mathrm{O} / \mathrm{THF} / \mathrm{MeOH} /$ r.t./14d/98\%; h) $\mathrm{Ac}_{2} \mathrm{O} / \mathrm{AcONa} /$ $80^{\circ} \mathrm{C} / 3 \mathrm{~h} ; \mathrm{Ac}_{2} \mathrm{O} /$ Pyridine $/ 80^{\circ} \mathrm{C} / 1 \mathrm{~h} / 96 \%$; i) $\left.\mathrm{Boc}_{2} \mathrm{O} / \mathrm{DMAP} / \mathrm{THF} / \mathrm{reflux} / 22.5 \mathrm{~h} ; \mathrm{j}\right) 28 \% \mathrm{NaOCH}_{3}$ in $\mathrm{MeOH} /$ $\mathrm{CH}_{2} \mathrm{Cl}_{2} / \mathrm{MeOH} /$ r.t./6h; k) Ac $2 \mathrm{O} /$ Pyridine/ $60^{\circ} \mathrm{C} / 2 \mathrm{~h} / 77 \%$; 1) $\mathrm{TMSN}_{3} / \mathrm{SnCl}_{4} / \mathrm{CHCl}_{3} /$ r.t./overnight $/ 99 \%$; m) $28 \% \mathrm{NaOCH}_{3}$ in $\mathrm{MeOH} / \mathrm{CH}_{2} \mathrm{Cl}_{2} / \mathrm{MeOH} /$ r.t./ quantitative yield; n) TEMPO/ $\mathrm{KBr} / \mathrm{NaOCl} /$ sat. aq. $\mathrm{NaHCO}_{3} / 4^{\circ} \mathrm{C} / 1 \mathrm{~d}$; o) 
658

659

660

661

662

663

664

665

666

667

668

669

670

671

672

673

674

675

676

677

678

679

680

681

682

683

684

685

686

687

688

689

690

691

692

693

694

2,2-dimethoxypropane/conc. $\mathrm{HCl} / \mathrm{MeOH} /$ r.t./ 1d; p) $\mathrm{Ac}_{2} \mathrm{O} / \mathrm{AcONa} / 60^{\circ} \mathrm{C} /$ overnight/ total yield $11 \%$ from compound 23; q) $\mathrm{Cu}(\mathrm{I}) \mathrm{Br} /$ sodium ascorbate in water/ $\mathrm{PMDETA} / \mathrm{MeOH}: \mathrm{CH}_{2} \mathrm{Cl}_{2}(1: 4, \mathrm{v} / \mathrm{v})$, distilled water/r.t./4 days; r) $\mathrm{CuSO} \cdot \mathrm{H}_{2} \mathrm{O} /$ sodium ascorbate in water/ $\mathrm{PMDETA} / \mathrm{THF} / 50^{\circ} \mathrm{C} /$ overnight; s) $\mathrm{CuSO}_{4} \cdot \mathrm{H}_{2} \mathrm{O}$ / sodium ascorbate in water/ PMDETA/THF $/ 50^{\circ} \mathrm{C} /$ overnight; t) $28 \% \mathrm{NaOCH}_{3}$ in $\mathrm{MeOH} / \mathrm{THF} / \mathrm{MeOH} /$ r.t./overnight; $\mathrm{u}$ ) $28 \% \mathrm{NaOCH}_{3}$ in $\mathrm{MeOH} /$ $\mathrm{THF} / \mathrm{MeOH} / \mathrm{CH}_{2} \mathrm{Cl}_{2} /$ r.t./overnight; trifluoroacetic acid/-20 ${ }^{\circ} \mathrm{C} / 1.2 \mathrm{~h}$; v) $0.0125 \mathrm{M} \mathrm{NaOH} / \mathrm{THF} / \mathrm{r}$.t./30 min/99\% yield

The synthesis of amino-functionalized cellobiose compound $\mathbf{1 0}$ from 2-propynyl (6-amino-6-deoxy- $\beta$-D-glucopyranosyl)-( $1 \rightarrow 4)$-6-amino-6-deoxy- $\beta$-D-glucopyranoside (18) was analogous to a method for a glucosamine derivative (Chen et al., 2010). Compound 10 was converted from compound 9 in 10 reaction steps. The $N$-acetyl groups at the C-6 positions of compound 19 would be relatively stable under alkali conditions. The amino groups of compound 18 are reactive and labile and are therefore protected by tert-butoxycarbonyl (Boc) groups. The primary hydroxy groups at the C-6 positions of compound $\mathbf{1 3}$ were tosylated with $p$-toluenesulfonyl chloride to give compound $\mathbf{1 4}$ in 51\% yield. The four secondary hydroxy groups of compound $\mathbf{1 4}$ were acetylated with acetic anhydride in pyridine to give compound $\mathbf{1 5}$ in $95 \%$ yield. The tosylate 15 was treated with sodium azide to give azide derivative $\mathbf{1 6}$ in 97\% yield via a nucleophilic substitution at the C-6 positions. Removal of the acetyl groups of compound $\mathbf{1 6}$ produced compound $\mathbf{1 7}$ in $87 \%$ yield. A Staudinger reaction afforded amino derivative $\mathbf{1 8}$ from azido compound 17 in 98\% yield. Compound 18 is an analogous derivative of a chitosan dimer. Because the reactivity and stability of compound 18, without protective groups for the amino and hydroxy groups, are unknown, the protected compound $\mathbf{1 0}$ was selected as the reactant for Huisgen 1,3-dipolar cycloaddition. Compound 18 was acetylated to give 6-(acetyl)amino derivative 19. Butoxycarbonylation of $N$-(acetyl)amino compound 19 followed by removal of the acetyl groups afforded 6-(Boc)amino derivative 21 via 6-acetyl(Boc)amino derivative 20. Compound 21 was acetylated to give 2-propynyl

(2,3,4-tri- $O$-acetyl-6-(Boc)amino-6-deoxy- $\beta$-D-glucopyranosyl)-(1 $\rightarrow 4)-2,3$-di- $O$-acetyl-6-(Boc)ami no-6-deoxy- $\beta$-D-glucopyranoside (10). Compound 10 is a precursor of the cationic hydrophilic segment that gives compound 2.

Compound 11 was prepared from cellobiose octaacetate (12) (H. Kamitakahara \& Nakatsubo, 2005 ) in five reaction steps and is a precursor of the anionic hydrophilic segment that gives compound 3. Compound $\mathbf{1 1}$ is a glycosyl azide derivative, although compounds $\mathbf{9}$ and $\mathbf{1 0}$ are 2-propenyl glycosides. The alkyne group was unstable under the reaction conditions for TEMPO oxidation of the primary alcohol. Methyl [(methyl

2,3,4-tri- $O$-acetyl- $\beta$-D-glucopyranosyluronate)-( $1 \rightarrow 4)$-2,3-di- $O$-acetyl- $\beta$-D-glucopyranosyl azide]uronate (11) was therefore chosen as the hydrophilic segment and treated with 2-propynyl tri-O-methyl-celluloside to produce compound 6. Cellobiose octaacetate (12) was converted into 2,3,4,6-tetra- $O$-acetyl- $\beta$-D-glucopyranosyl-( $(\rightarrow 4)$-2,3,6-tri- $O$-acetyl- $\beta$-D-glucopyranosyl azide (22) 
695 (H. Kamitakahara \& Nakatsubo, 2005). Removal of the acetyl groups of compound 22 gave

$696 \beta$-D-glucopyranosyl-( $1 \rightarrow 4)$ - $\beta$-D-glucopyranosyl azide (23). TEMPO oxidation of cellobiosyl azide

69723 gave $(\beta$-D-glucopyranuronosyl)-( $1 \rightarrow 4)-\beta$-D-glucopyranuronosyl azide $(\mathbf{2 4})$. The uronic acids of

698 compound $\mathbf{2 4}$ were esterified to give methyl [(methyl

$699 \beta$-D-glucopyranosyluronate)-(1 $\rightarrow 4)-\beta$-D-glucopyranosyl azide]uronate $(\mathbf{2 5})$. The remaining hydroxy 700 groups of compound $\mathbf{2 5}$ were acetylated to give methyl [(methyl

701 2,3,4-tri- $O$-acetyl- $\beta$-D-glucopyranosyluronate)-( $1 \rightarrow 4)-2,3$-di- $O$-acetyl- $\beta$-D-glucopyranosyl 702 azide]uronate (11).

703 Figure 1A shows ${ }^{1} \mathrm{H}-\mathrm{NMR}$ spectra of cellobiose derivatives 9, 10, and 11. Figure $\mathrm{S} 1$ in the 704 Supporting Information show ${ }^{13} \mathrm{C}-\mathrm{NMR}$ spectra of cellobiose derivatives $\mathbf{9}, \mathbf{1 0}$, and 11. 
A
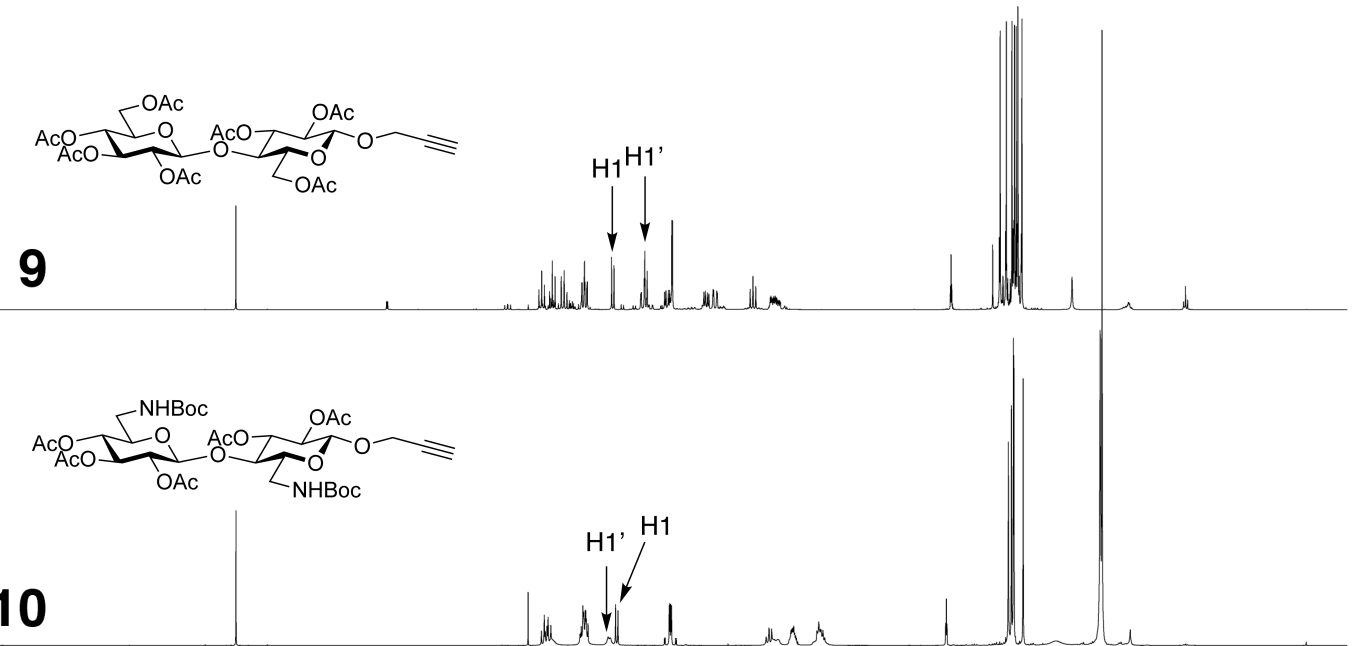

${ }_{\mathrm{OAc}}^{\mathrm{NHBoc}}$

10

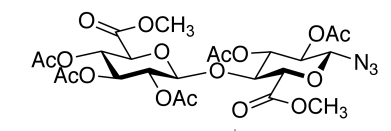

11
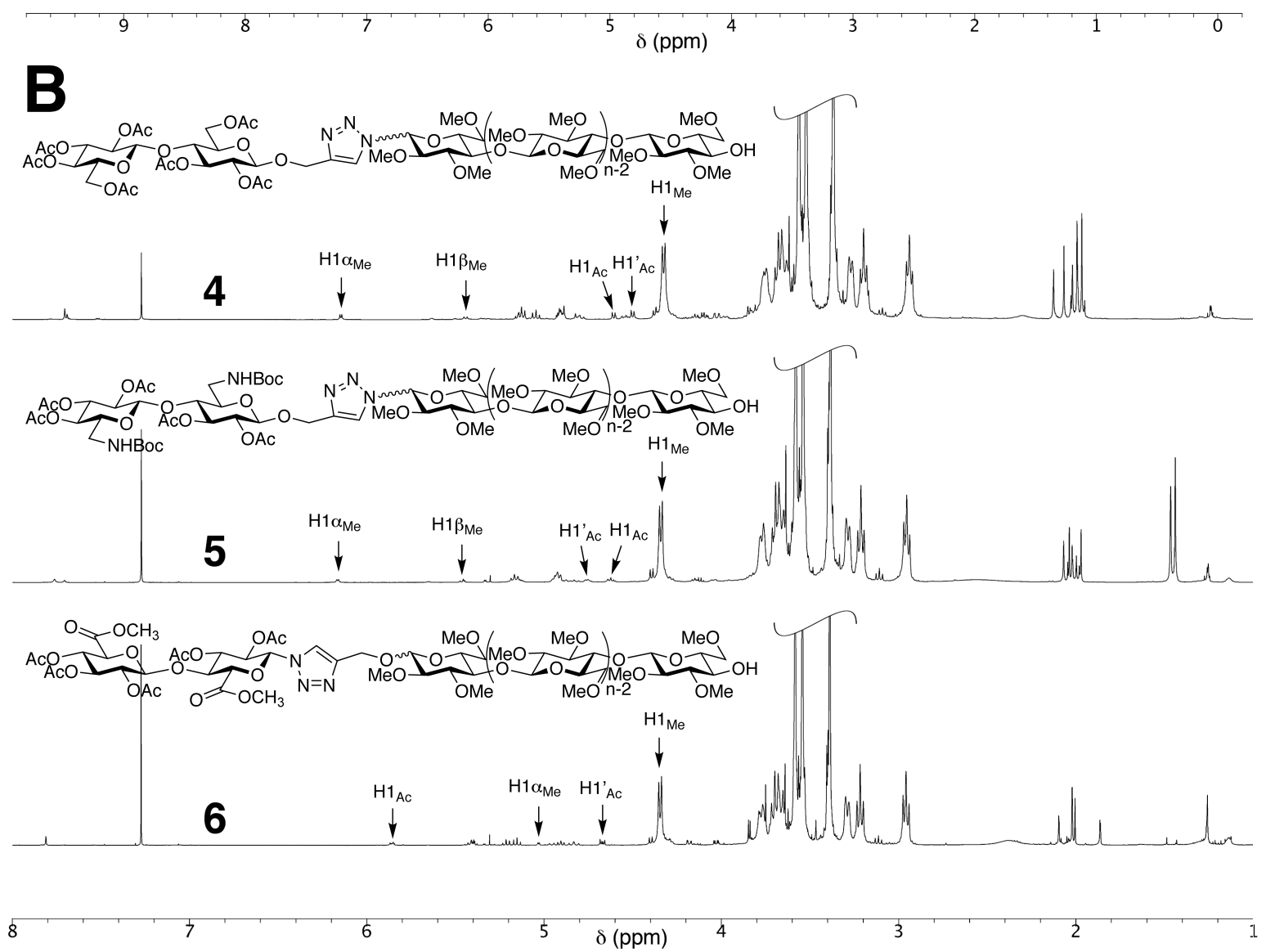

705 Figure 1. ${ }^{1} \mathrm{H}-\mathrm{NMR}$ spectra of (A) cellobiose derivatives $\mathbf{9}, \mathbf{1 0}$, and 11, and (B) compounds 4, 5, 706 and 6 after $\mathrm{CuAAC}$ reaction 


\subsubsection{Synthesis of trehalose-type diblock methylcellulose analogues $\mathbf{1}, \mathbf{2}$, and $\mathbf{3}$}

The Huisgen 1,3-dipolar cycloaddition followed by removal of the protective groups afforded the nonionic, cationic, and anionic diblock methylcellulose analogues 1-(tri- $O$-methyl-cellulosyl)-4-( $\beta$-D-glucopyranosyl-( $1 \rightarrow 4)-\beta$-D-glucopyranosyloxymethyl)-1H-1,2,3 -triazole (1), 1-(tri-O-methyl-cellulosyl)-4-(6-amino-6-deoxy- $\beta$-D-glucopyranosyl-( $1 \rightarrow 4)-6$-amino-6-deoxy- $\beta$-Dglucopyranosyloxymethyl)-1 $H$-1,2,3-triazole (2), and 4-(tri-O-methyl-cellulosyloxylmethyl)-1-( $\beta$-D-glucopyranuronosyl-( $1 \rightarrow 4)$-D-glucopyranuronosyl)-1 $H$-1,2,3-triazole (3), as shown in Scheme 1.

The Huisgen 1,3-dipolar cycloaddition between the alkyne and azido derivatives was successfully carried out. An excess amount, three equivalents, of cellobiose derivatives 9, 10, and 11 relative to polymeric methylcellulose derivatives $\mathbf{7}$ and $\mathbf{8}$ produced trehalose-type diblock methylcellulose derivatives $\mathbf{4 , 5}$, and $\mathbf{6}$ with no remaining $\mathbf{7}$ and $\mathbf{8}$. In the MALDI-TOF mass spectra shown in Figure S2, we observed pseudo-molecular-ion peaks for compounds 4, 5, and 6 (see the Supporting Information), which means that complete end-functionalization of the methylcellulose derivatives was carried out via the Huisgen 1,3-dipolar cycloaddition.

Figure 1B shows the ${ }^{1} \mathrm{H}-\mathrm{NMR}$ spectra of compounds 4,5 , and $\mathbf{6}$ obtained as a result of the Huisgen 1,3-dipolar cycloadditions. The proton resonances of the cellobiose segments were small relative to those of the polymeric methylcelluloses. The triazole ring proton, however, appeared at approximately 7.7-7.8 ppm, which enabled us to confirm the successful formation of the trehalose-type diblock methylcellulose analogues. The ${ }^{1} \mathrm{H}-\mathrm{NMR}$ spectrum of compound 4 revealed that the $\mathrm{C}-1$ proton of the $\alpha$ - and $\beta$-anomers of the methylcellulosyl residue appeared at 6.15 and $5.44 \mathrm{ppm}$, respectively. The $\alpha / \beta$ ratio was approx. $2 / 1$. Two triazole protons appeared in the

\footnotetext{
${ }^{1} \mathrm{H}-\mathrm{NMR}$ spectra of compounds $\mathbf{4}$ and $\mathbf{5}$, which means that the $\alpha$ - and $\beta$-anomers of the
} methylcellulosyl residue affected the chemical shift of those 1,2,3-triazole protons. In contrast, one triazole proton appeared in the ${ }^{1} \mathrm{H}-\mathrm{NMR}$ spectra of compound $\mathbf{6}$. The proton resonance corresponding to the anomeric center of the methyl glucopyranosiduronate residue attached to the triazole unit appeared at approx. 5.8-5.9 ppm as a doublet. The anomeric center at the reducing end of the methylcelluloside appeared at $5.03 \mathrm{ppm}$ as a doublet $(J=3.5 \mathrm{~Hz})$. After the removal of the acyl groups of compounds 4, 5, and 6 , the proton resonances of the unmodified cellobiosyl residues of compounds $\mathbf{1}, \mathbf{2}$, and $\mathbf{3}$ overlapped with those of methylcellulose (data not shown). Therefore, we were unable to conclude from the results of the NMR analysis that compounds 1, 2, and $\mathbf{3}$ were obtained. However, the MALDI-TOF mass spectra of compounds $\mathbf{1 , 2}$, and 3 proved that we succeeded in establishing synthetic routes for these target compounds (Figure 
742

743

744

745

746

S2). The MALDI-TOF mass spectrum of compound 1 shows that the pseudo-molecular-ion peaks of compound 1 appeared as sodium adducts. In contrast, the MALDI-TOF mass spectrum of compound $\mathbf{2}$ revealed that amino derivative $\mathbf{2}$ was observed as a proton adduct.

The synthesis of compound $\mathbf{3}$ from compound $\mathbf{6}$ under alkali conditions did not remove the a proton (H-5) of the C-6 carbonyl carbon atom of the hydrophilic segment to promote $\beta$-elimination; therefore, there was no depolymerization of a glucuronic acid at the non-reducing end of the hydrophilic segment of compound $\mathbf{3}$. We did not observe any evidence of the $\beta$-elimination, as shown in Figure 1, although there are unassigned peaks. Purity of compounds 1, 2, and 3 was confirmed by means of MALDI-TOF MS, GPC after acetylation, and analytical thin layer chromatography (TLC).

In addition, the zeta potential of compounds 1, 2, and $\mathbf{3}$ revealed that compounds $\mathbf{2}$ and $\mathbf{3}$ involve cationic and anionic functional groups, respectively, as summarized later in Table 1. The zeta potential data also proved that compounds $\mathbf{1 , 2}$, and $\mathbf{3}$ were produced.

\subsection{Physical properties of trehalose-type diblock methylcellulose analogues 1,2 , and 3}

Some physical properties of compounds $\mathbf{1 , 2}$, and $\mathbf{3}$ are summarized in Table 1. We investigated the properties of the aqueous solutions. Nonionic compound 1 shows a negative zeta potential (-6.8 $\mathrm{mV}$ ), likely because oxygen atoms along the methylcellulose residue affect the negative charge for the whole molecule. The zeta potential of cationic compound 2 was slightly higher than that of nonionic compound $\mathbf{1}$. Two amino groups at the end of molecule $\mathbf{2}$ affected the total zeta potential of compound $\mathbf{2}$ in water. The zeta potential of compound $\mathbf{3}$ was the lowest among compounds $\mathbf{1 , 2}$, and 3, which means that the carboxylic acid at the end of the molecule affected the overall zeta potential of compound 3. Table 1 also summarizes the interfacial properties, DLS data of the aqueous solutions, DSC data, and gelation properties.

Table 1. Physicochemical properties of compounds 1, 2, and $\mathbf{3}$

\begin{tabular}{|c|c|c|c|c|c|c|c|c|}
\hline \multirow[b]{2}{*}{$\begin{array}{l}\text { Compound } \\
\text { no. }\end{array}$} & \multirow{2}{*}{$\begin{array}{c}\text { Zeta potential }(\mathrm{mV}) \\
0.2 \mathrm{wt} \%, 35^{\circ} \mathrm{C}\end{array}$} & \multicolumn{2}{|c|}{ Interfacial property } & \multirow{2}{*}{$\begin{array}{c}\text { Aggregation } \\
\text { temperature }\left({ }^{\circ} \mathrm{C}\right) \\
\text { judged by DLS } \\
(0.2 \mathrm{wt} \%) \\
\end{array}$} & \multicolumn{2}{|c|}{ Thermal property detected by DSC (4.0 wt $\%)$} & \multicolumn{2}{|c|}{ Gelation property } \\
\hline & & $\begin{array}{c}\text { Critical Micelle } \\
\text { Concentration }(\mathrm{CMC}) \\
(\mathrm{mg} / \mathrm{mL})\end{array}$ & $\begin{array}{l}\text { Surface tention } \\
\text { at } \mathrm{CMC}(\mathrm{mN} / \mathrm{m})\end{array}$ & & $\begin{array}{c}\text { Endothermic } \\
\text { peak }\left({ }^{\circ} \mathrm{C}\right) \\
\end{array}$ & $\begin{array}{l}\text { Exothermic } \\
\text { peak }\left({ }^{\circ} \mathrm{C}\right)\end{array}$ & $2.0 w t \%$ & $4.0 \mathrm{wt} \%$ \\
\hline 1 & -6.8 & $6.5 \times 10^{-3}$ & 48.2 & 33 & 29 & 5 & $+\left(30^{\circ} \mathrm{C}\right)$ & $+\left(30^{\circ} \mathrm{C}\right)$ \\
\hline 2 & -3.9 & $2.6 \times 10^{-3}$ & 44.0 & 34 & 33 & 8 & - & $+\left(35^{\circ} \mathrm{C}\right)$ \\
\hline 3 & -28.2 & $3.5 \times 10^{-3}$ & 44.3 & $20-29$ & 24 & 3 & - & $+\left(30^{\circ} \mathrm{C}\right)$ \\
\hline
\end{tabular}

\subsubsection{Surface activity of aqueous solutions of compounds $\mathbf{1}, \mathbf{2}$, and $\mathbf{3}$}

The trehalose-type diblock methylcellulose analogues 1, 2, and $\mathbf{3}$ exhibited amphiphilic properties and better surface activities than commercially available methylcellulose SM-4, as shown in Figure 2. The surface activity was in the order $\mathbf{2}>\mathbf{3}>\mathbf{1}$. In particular, cationic compound $\mathbf{2}$ exhibited the best surface activity among the compounds tested, likely because its diblock structure, comprising a 
775

776

777

778

779

780

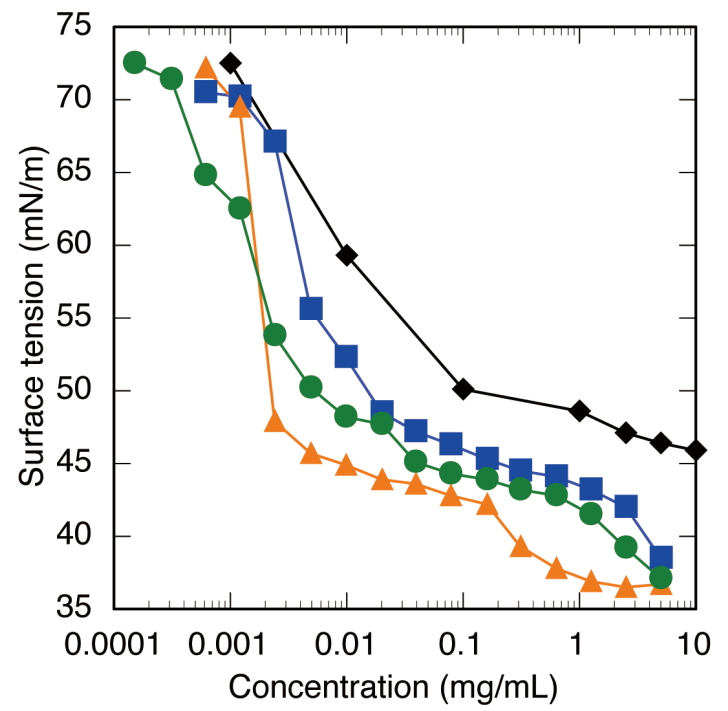

781 Figure 2. Surface tension-concentration curves of compounds 1, 2, and 3; blue solid square: 1; 782 orange solid triangle: $\mathbf{2}$; green solid circle: $\mathbf{3}$; black solid diamond: commercially available 783 methylcellulose SM-4.

784
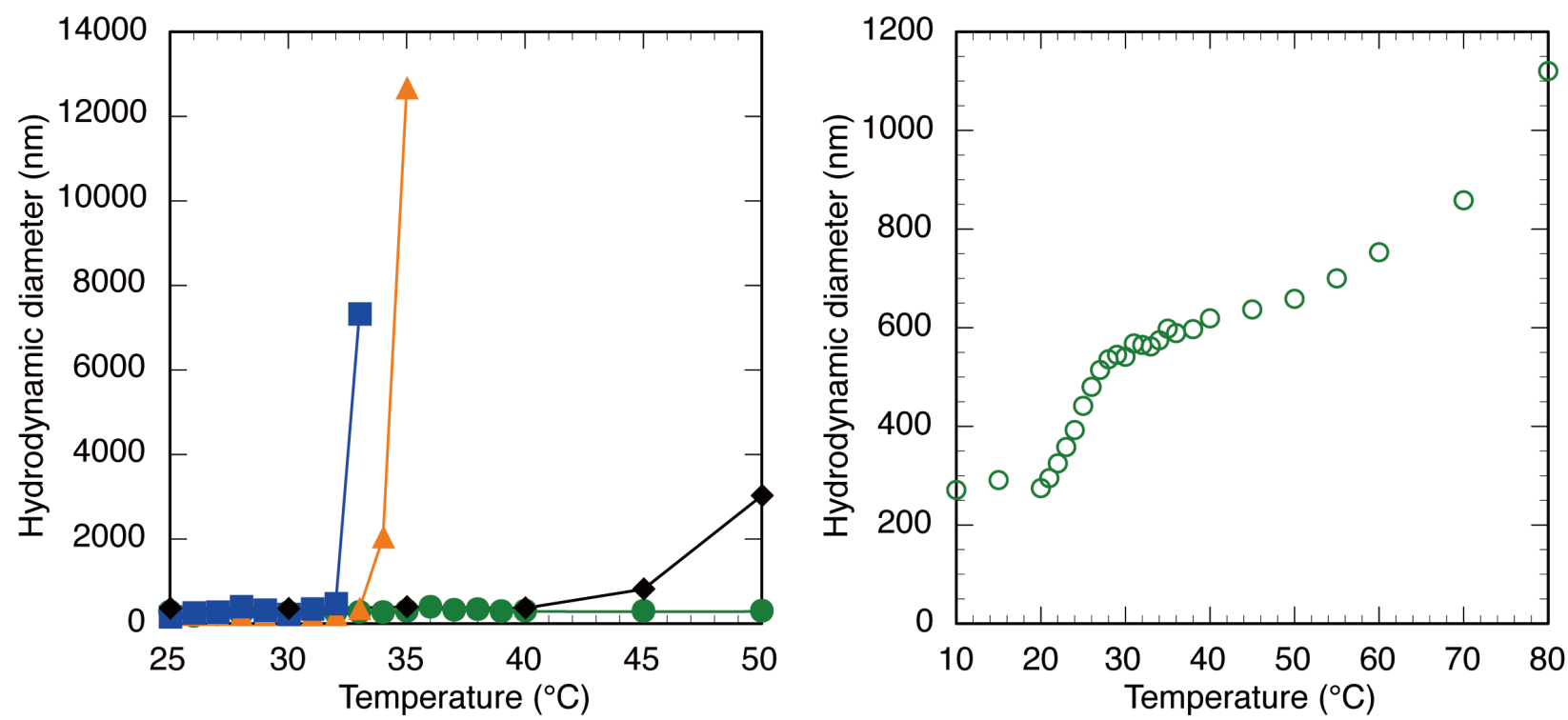

Figure 3. Hydrodynamic diameter of 0.2 wt.\% aq. solution of compounds 1, 2, and 3 (a) and expanded graph of $2.0 \mathrm{wt} . \%$ aq. solution of compound 3 (b) as a function of temperature; blue solid square: 1; orange solid triangle: 2; green solid circle: 3; black solid diamond: commercially available methylcellulose SM-4. 
3.2.2. Thermoresponsive aggregation behavior of compounds $\mathbf{1}, \mathbf{2}$, and $\mathbf{3}$ in water

791 The aggregation properties of compounds $\mathbf{1}, \mathbf{2}$, and $\mathbf{3}$ in $0.2 \mathrm{wt}$ \% aqueous media were different, as 792 shown in Figure 3. Compounds $\mathbf{1}$ and $\mathbf{2}$ suddenly aggregated at $33{ }^{\circ} \mathrm{C}$ and $34{ }^{\circ} \mathrm{C}$, respectively, but 793 compound 3 did not show obvious aggregation properties. In contrast, the $2.0 \mathrm{wt}$ \% aqueous 794 solution of compound 3 gradually aggregated in the range from 20 to $29^{\circ} \mathrm{C}$, likely because its 795 relatively large negative zeta potential would inhibit its self-aggregation behavior. The hydrophilic 796 segments affected molecular aggregation, with the result that the nonionic, cationic, and anionic 797 compounds exhibited different thermoresponsive temperatures.

\subsubsection{Thermal properties of aqueous solutions of compounds $\mathbf{1}, \mathbf{2}$, and $\mathbf{3}$}

800 Figure 4 shows DSC data for aqueous solutions of compounds 1, 2, and $\mathbf{3}$. Nonionic compound 1 801 and cationic compound 2 exhibited endothermic peaks at $29^{\circ} \mathrm{C}$ and $33{ }^{\circ} \mathrm{C}$, respectively. In contrast, 802 anionic compound 3 exhibited a broad endothermic peak in the range from 13 to $30{ }^{\circ} \mathrm{C}$. The 803 endothermic peak indicated dehydration surrounding the methylcellulose analogues. The results of 804 DLS experiments are consistent with those of DSC analysis, indicating that a dehydration process 805 followed by self-aggregation occurred. For instance, the endothermic peak of the aqueous solution 806 of compound 1 appeared at $29^{\circ} \mathrm{C}$ upon heating (heating rate: $3.5^{\circ} \mathrm{C} / \mathrm{min}$ ). After the endothermic 807 temperature of compound 1 at $29{ }^{\circ} \mathrm{C}$ was detected by DSC measurements, the aggregation of 808 compound 1 was apparently observed by DLS measurements at $33{ }^{\circ} \mathrm{C}$, as summarized in Table 1 . 809 The same tendency was also observed for compound 2. The dehydration of compound $\mathbf{3}$ occurred 810 slowly, likely because the large negative zeta potential of compound $\mathbf{3}$ would disturb the 811 intermolecular aggregation process.

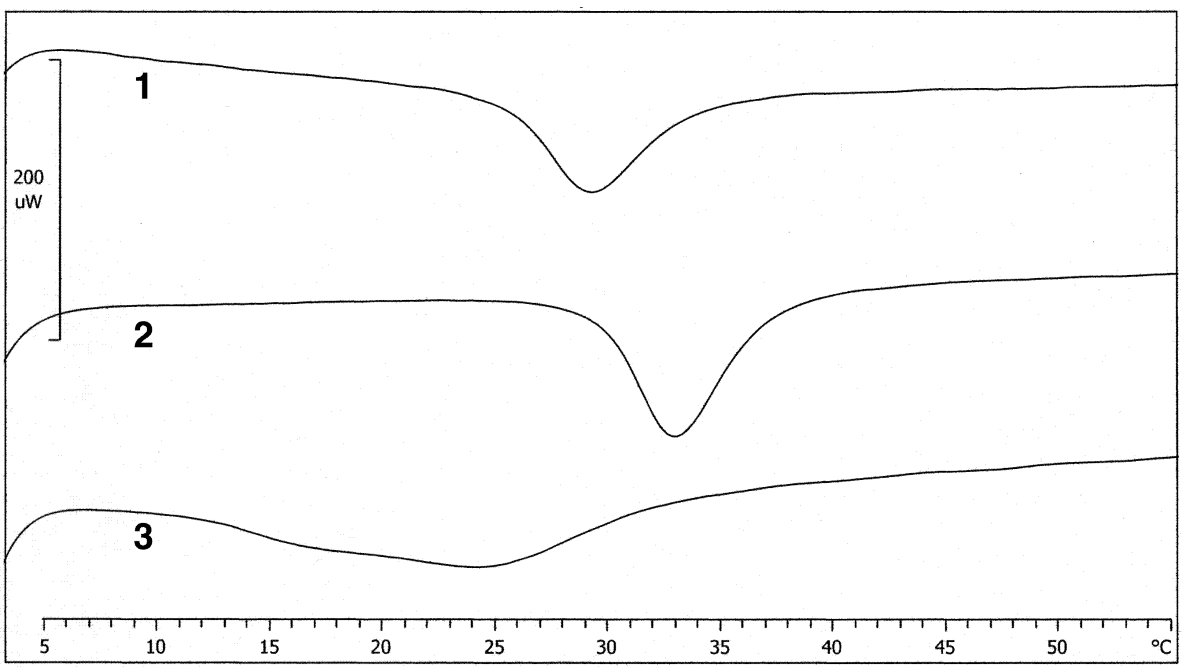

814 Figure 4. DSC thermograms of $2.0 \mathrm{wt}$ \% aqueous solutions of compounds $\mathbf{1}, \mathbf{2}$, and $\mathbf{3}$. Heating rate: $3.5^{\circ} \mathrm{C} / \mathrm{min}$. 
3.3. Observation of thermoresponsive supramolecular hydrogels comprising compounds 1,2 , and 3

819 3.3.1. Visual observation of thermoresponsive supramolecular hydrogels

820 Figure 5 shows photographs of dispersions of compounds $\mathbf{1}, \mathbf{2}$, and $\mathbf{3}$ in water at 0,30 , and $35^{\circ} \mathrm{C}$.

821 With increasing temperature, the trehalose-type methylcellulose analogues self-aggregated,

822 triggered by dehydration around the molecules. The multi-molecular assembly of the trehalose-type 823 methylcellulose analogues caused a macroscopic change from sol to hydrogel. A 2 wt. \% aqueous

824 solution of nonionic compound 1 became a hydrogel at $30^{\circ} \mathrm{C}$. In contrast, 2 wt. \% aqueous

825 solutions of cationic compound 2 and anionic compound 3 stayed in the sol state. However, 4 wt. \% 826 aqueous solutions of ionic compounds 2 and $\mathbf{3}$ became hydrogels at 35 and $30^{\circ} \mathrm{C}$, respectively. A 4 827 wt. \% aqueous solution of nonionic compound 1 also became a hydrogel at $30{ }^{\circ} \mathrm{C}$.

828

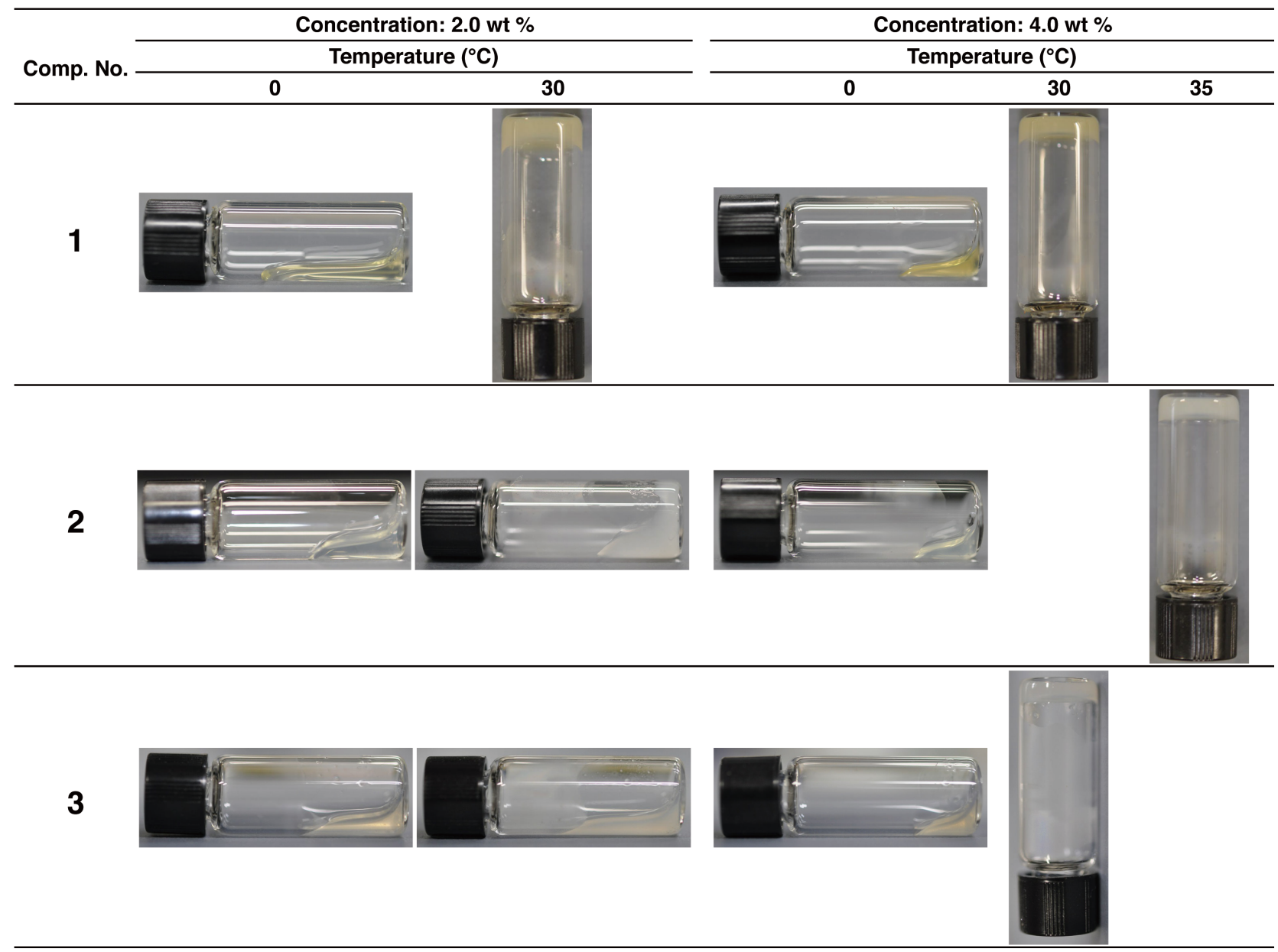

830 Figure 5. Photographs of dispersion of compounds 1, 2, and 3 in water at 0,30 , and $35^{\circ} \mathrm{C}$

832 3.3.2. Layered structure of lyophilized hydrogels from compounds $\mathbf{1}, \mathbf{2}$ and $\mathbf{3}$.

833 Scanning electron microscopy images are shown in Figure $6 \mathrm{a}-\mathrm{f}$. The sections of the three kinds of 
834 hydrogels show layered structures (Figure 6, insets b, d, and f). Short, regularly arranged

835 protuberances can be seen on one side of the layers (Figure $6 \mathrm{a}, \mathrm{c}$ and e). The surfaces of the layers

836 in the hydrogel from cationic compound $\mathbf{2}$ were very smooth relative to those in the hydrogels from

837 compounds $\mathbf{1}$ and $\mathbf{3}$, which suggests that the chemical structure of the hydrophilic segments affects

838 the structure of the hydrogels.

839
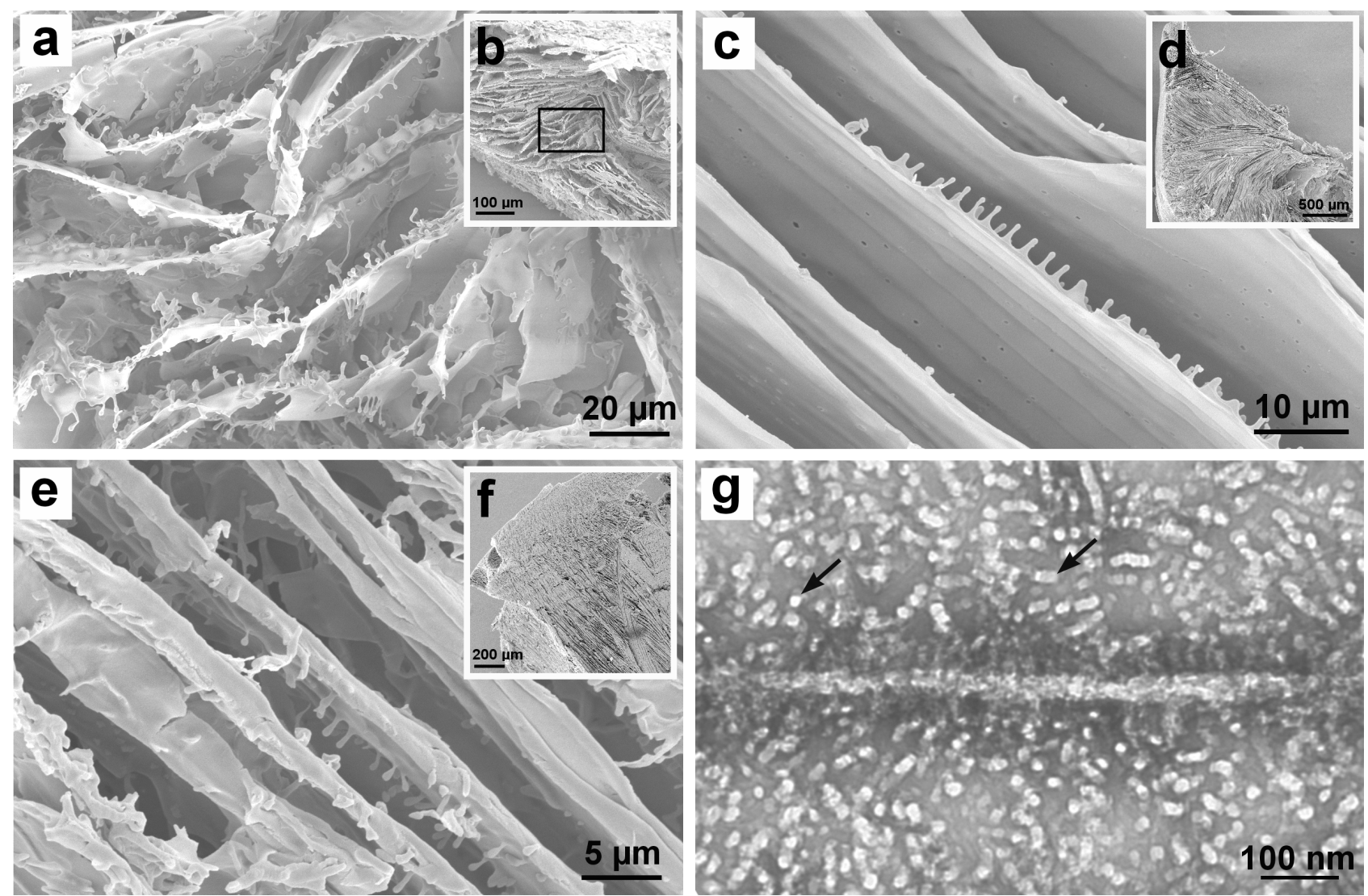

840 Figure 6. Scanning electron microscopic images of hydrogels from compounds 1 (a, b), 2 (c, d), and

8413 (e, f); a transmission electron microscopic image of compound 1 (g). a, c, e were enlarged images

842 from low magnified images (b, $\mathrm{d}$ and $\mathrm{f}$ ). Arrows in $\mathrm{g}$ indicate square and rectangular aggregated 843 particles.

3.3.3. Ultrastructures of thermoresponsive supramolecular hydrogel of compound $\mathbf{1}$

A transmission electron microscopy image is shown in Figure $6 \mathrm{~g}$. Square or rectangular structures of approximately $20 \mathrm{~nm} \times 20-55 \mathrm{~nm}$ (Figure $6 \mathrm{~g}$, arrows) can be seen. In addition, a long linear structure of approximately $20 \mathrm{~nm}$ width with short fine protuberances on both sides was observed.

849 The molecular length of compound 1 is approx. $10 \mathrm{~nm}$. Two molecules of compound 1 exist in the

$85020 \mathrm{~nm}$ width for a short side of a rectangular structure. We propose a hypothesis for how the

851 molecules of compound 1 self-assembled to form square or rectangular structures in Figure S3 in the Supporting Information. The thickness of those structures was approximately $1.2 \mathrm{~nm}$, as 
853

854

855

856

confirmed by atomic force microscopy (data not shown). The long side of the rectangular structure alters depending on the particles. Hydrophobic interactions between tri- $O$-methylcellulose segments would elongate the long side of the rectangular structure upon heating. Hydrogen bonding between hydrophilic segments would drive self-assembly between square and/or rectangular structures to form a linear structure over $1 \mu \mathrm{m}$. This combined structure would grow into a shish-kebab-like supramolecular structure. Finally those shish-kebab-like supramolecular structures would grow into a two-dimensional sheet structure, as shown in Figure 6 a.

\section{Conclusion}

We succeeded in the methycellulose analogues end-functionalized with nonionic and ionic cellobiose derivatives via Huisgen 1,3-dipolar cycloaddition. New trehalose-type diblock methylcellulose analogues, nonionic $\mathbf{1}$, cationic $\mathbf{2}$, and anionic $\mathbf{3}$, provide understanding of the detailed structure-property relationships of cellulose ether derivatives. The synthetic routes for them were shortened, relative to those we have already reported (Nakagawa et al., 2012). The methodology described in this paper allows us to synthesize a variety of diblock methylcellulose analogues with a series of hydrophilic segments, thereby developing new applications of cellulose derivatives. Cationic compound $\mathbf{2}$ exhibited higher surface activity than anionic compound $\mathbf{3}$ and nonionic compound $\mathbf{1}$. The two amino groups at the end of the trehalose-type diblock methylcellulose analogue affected its self-assembly behavior at the interface between water and air. Not only nonionic 1 but also cationic 2 and anionic 3 formed thermoresponsive supramolecular hydrogels in water at under $37^{\circ} \mathrm{C}$, close to human body temperature. This fact means that the methylcellulose-based hydrogels including a nonionic or ionic cellobiosyl segment would respond to human body temperature and are comparable with those based on poly( $N$-isopropyl acrylamide) (Ashraf, Park, Park, \& Lee, 2016). These methylcellulose-based new materials will be applicable for the similar uses as poly( $N$-isopropyl acrylamide). Trehalose-type methylcellulose analogues from natural resource would produce eco-friendly surfactant, and safe thermoresponsive hydrogel matrices for drug release.

\section{Acknowledgements}

This work was in part supported by Grants-in-Aid for Scientific Research (B) nos. 24380092 and $15 \mathrm{H} 04531$.

\section{Figure Legends}

Scheme 1. Synthetic routes for cellobiose derivatives

Figure 1. ${ }^{1} \mathrm{H}-\mathrm{NMR}$ spectra of (A) cellobiose derivatives $\mathbf{9}, \mathbf{1 0}$, and 11, and (B) compounds 4, 5, and 6 after $\mathrm{CuAAC}$ reaction 
889

890

891

892

893

894

895

896

897

898

899

900

901

902

903

904

905

906

907

908

909

910

911

912

913

914

915

916

917

918

919

920

921

922

923

924

Figure 2. Surface tension-concentration curves of compounds 1, 2, and 3; blue solid circle: 1;

orange solid circle: $\mathbf{2}$; green solid circle: $\mathbf{3}$; black solid circle: commercially available methylcellulose SM-4.

Figure 3. Hydrodynamic diameter of $0.2 \mathrm{wt} . \%$ aq. solution of compounds 1, 2, and 3 (a) and expanded graph of $2.0 \mathrm{wt} . \%$ aq. solution of compound 3 (b) as a function of temperature; blue solid circle: 1; orange solid circle: 2; green solid circle: 3; black solid circle: commercially available methylcellulose SM-4.

Figure 4 . DSC thermograms of 2.0 wt. $\%$ aqueous solutions of compounds $\mathbf{1}, \mathbf{2}$, and $\mathbf{3}$. Heating rate: $3.5^{\circ} \mathrm{C} / \mathrm{min}$.

Figure 5. Photographs of dispersion of compounds 1, 2, and 3 in water at 0,30 , and $35^{\circ} \mathrm{C}$

Figure 6. Scanning electron microscopic images of hydrogels from compounds $\mathbf{1}(\mathrm{a}, \mathrm{b}), \mathbf{2}$ (c, d), and 3 (e, f); a transmission electron microscopic image of compound $\mathbf{1}(\mathrm{g})$. a, c, e were enlarged images from low magnified images (b, $\mathrm{d}$ and $\mathrm{f}$ ). Arrows in $\mathrm{g}$ indicate square and rectangular aggregated particles.

Table 1. Physicochemical properties of compounds 1, 2, and $\mathbf{3}$

Figure S1. ${ }^{13} \mathrm{C}$-NMR spectra of cellobiose derivatives 9, 10, and $\mathbf{1 1}$

Figure S2. MALDI-TOF MS spectra of compounds after CuAAC reaction and of compounds $\mathbf{1}, \mathbf{2}$, and $\mathbf{3}$ after removal of protective groups

Figure S3. Schematic figure of self-assembly process of compound 1 upon heating

Red hexagon: 2,3,6-tri-O-methyl glucose residue; Blue hexagon: unmodified glucose residue

\section{References}

Ashraf, S., Park, H.-K., Park, H., \& Lee, S.-H. (2016). Snapshot of phase transition in thermoresponsive hydrogel PNIPAM: Role in drug delivery and tissue engineering. Macromol. Res., 24(4), 297-304.

Chen, Q., Bian, N., Cao, C., Qiu, X. L., Qi, A. D., \& Han, B. H. (2010). Glucosamine hydrochloride functionalized tetraphenylethylene: A novel fluorescent probe for alkaline phosphatase based on the aggregation-induced emission. Chemical Communications, 46(23), 4067-4069.

Heinze, T., Erler, U., Nehls, I., \& Klemm, D. (1994). Determination of the substituent pattern of heterogeneously and homogeneously synthesized carboxymethyl cellulose by using high-performance liquid chromatography. Angew. Makromol. Chem., 215, 93-106.

Hossel, P., Dieing, R., Norenberg, R., Pfau, A., \& Sander, R. (2000). Conditioning 
925

926

927

928

929

930

931

932

933

934

935

936

937

938

939

940

941

942

943

944

945

946

947

948

949

950

951

952

953

954

955

956

957

958

959

960

polymers in today's shampoo formulations - efficacy, mechanism and test methods. Int. J. Cosmet. Sci., 22(1), 1-10.

Isogai, A., \& Kato, Y. (1998). Preparation of polyuronic acid from cellulose by TEMPO-mediated oxidation. Cellulose (London), 5(3), 153-164.

Kamitakahara, H., \& Nakatsubo, F. (2005). Synthesis of diblock copolymers with cellulose derivatives. 1. Model study with azidoalkyl carboxylic acid and cellobiosylamine derivative. Cellulose, 12(2), 209-219.

Kamitakahara, H., Nakatsubo, F., \& Klemm, D. (2006). Block co-oligomers of tri-O-methylated and unmodified cello-oligosaccharides as model compounds for methylcellulose and its dissolution/gelation behavior. Cellulose, 13(4), 375-392.

Kamitakahara, H., Suhara, R., Yamagami, M., Kawano, H., Okanishi, R., Asahi, T., \& Takano, T. (2016). A versatile pathway to end-functionalized cellulose ethers for click chemistry applications. Carbohydrate Polymers, 151, 88-95.

Kato, T., Yokoyama, M., \& Takahashi, A. (1978). Melting temperatures of thermally reversible gels. Colloid and Polymer Science, 256, 15-21.

Moni, L., Marra, A., Skotnicki, J. S., Koehn, F. E., Abou-Gharbia, M., \& Dondoni, A. (2013). Synthesis of rapamycin glycoconjugates via a CuAAC-based approach. Tetrahedron Lett., 54(51), 6999-7003.

Nakagawa, A., Fenn, D., Koschella, A., Heinze, T., \& Kamitakahara, H. (2011a). Physical Properties of Diblock Methylcellulose Derivatives with Regioselective Functionalization Patterns: First Direct Evidence that a Sequence of 2,3,6-Tri-O-methyl-glucopyranosyl Units Causes Thermoreversible Gelation of Methylcellulose. Journal of Polymer Science Part B-Polymer Physics, 49(21), 1539-1546.

Nakagawa, A., Fenn, D., Koschella, A., Heinze, T., \& Kamitakahara, H. (2011b). Synthesis of Diblock Methylcellulose Derivatives with Regioselective Functionalization Patterns. Journal of Polymer Science Part A-Polymer Chemistry, 49(23), 4964-4976.

Nakagawa, A., Kamitakahara, H., \& Takano, T. (2012). Synthesis and thermoreversible gelation of diblock methylcellulose analogues via Huisgen 1,3-dipolar cycloaddition. Cellulose, 19(4), 1315-1326.

Rees, D. A. (1972). Polysaccharide gels, A molecular view. Chem. \& Ind. (London), 19, 630-636.

Rinaudo, M. (2006). Chitin and chitosan: properties and applications. Prog. Polym. Sci., 31(7), 603-632.

Saito, T., Kimura, S., Nishiyama, Y., \& Isogai, A. (2007). Cellulose Nanofibers 
Prepared by TEMPO-Mediated Oxidation of Native Cellulose.

963 Saito, T., Nishiyama, Y., Putaux, J.-L., Vignon, M., \& Isogai, A. (2006). Homogeneous 964 Suspensions of Individualized Microfibrils from TEMPO-Catalyzed Oxidation of 965 Native Cellulose. Biomacromolecules, 7(6), 1687-1691.

966

Savage, A. B. (1957). Temperature-Viscosity Relationships for Water-Soluble Cellulose Ethers. Industrial and Engineering Chemistry, 49, 99.

968 Schamann, M., \& Schafer, H. J. (2003). TEMPO-mediated anodic oxidation of methyl 969 glycosides and 1-methyl and 1-azido disaccharides. Eur. J. Org. Chem.(2), 971 Teshirogi, T., Yamamoto, H., Sakamoto, M., \& Tonami, H. (1979). Synthesis of 972 6-amino-6-deoxycellulose. Sen'i Gakkaishi, 35(12), T525-T529. 351-358. 